COMPUTATIONAL METHODS IN APPLIED MATHEMATICS , Vol. 9 (2009), No. 4, pp. _-_-_(In Press)

(C) 2009 Institute of Mathematics of the National Academy of Sciences of Belarus

\title{
Numerical Solution of a Non-Local Problem Modelling Ohmic Heating of Foods
}

\author{
C. V. Nikolopoulos \\ Department of Mathematics, University of the Aegean \\ Karlovasi,Samos, 83200, Greece. \\ E-mail: cnikolo@aegean.gr
}

\begin{abstract}
An upwind and a Lax-Wendroff scheme are introduced for the solution of a one dimensional non-local problem modelling Ohmic heating of Foods. The schemes are studied regarding their consistency, stability and the rate of convergence for the cases that the problem attains a global solution in time. A high resolution scheme is also introduced and it is shown that it is total-variation-stable. Finally some numerical experiments are presented in support of the theoretical results.
\end{abstract}

Keywords: Non-local hyperbolic problems, Finite Difference Schemes, Upwind Method, Lax-Wendroff Method, Ohmic Heating.

\section{Introduction}

We consider the non-local initial boundary value problem,

$$
\begin{gathered}
u_{t}(x, t)+u_{x}(x, t)=\lambda \frac{f(u(x, t))}{\left(\int_{0}^{1} f(u(x, t)) d x\right)^{2}}, \quad 0<x<1, \quad t>0, \\
u(0, t)=0, \quad t>0, \\
u(x, 0)=u_{0}(x) \geqslant 0, \quad 0<x<1,
\end{gathered}
$$

where $\lambda>0$. The function $u(x, t)$ represents the dimensionless temperature when an electric current flows through a conductor (e.g. food) with temperature dependent on electrical resistivity $f(u)>0$, subject to a fixed potential difference $V>0$. The (dimensionless) resistivity $f(u)$ may be either an increasing or a decreasing function of temperature depending strongly on the type of the material (food). Problem (1.1) models one of the main methods for sterilizing food. The sterilization can take place by electrically heating the food rapidly. The food is passed through a conduit, part of which lies between two electrodes. A high electric current flowing between the electrodes results in Ohmic heating of the food which quickly gets hot. This procedure can be modelled by problem (1.1). A detailed derivation of the model, (1.1), can be found in [15].

The problem was considered initially in [19] where the stability of models allowing for different types of flow is studied. More background on this type of process can be found in $[5,4,9,20,22,24]$. In [15] problem (1.1) is also studied and it is found that for $f$ decreasing with $\int_{0}^{\infty} f(s) d s<\infty$ then blow-up occurs if the parameter $\lambda\left(\propto V^{2}\right)$ is too 
large for a steady state to exist or if the initial condition is too big. If $f$ is increasing with $\int_{0}^{\infty} d s / f(s)<\infty$ blow-up is also possible. If $f$ is increasing with $\int_{0}^{\infty} d s / f(s)=\infty$ or decreasing with $\int_{0}^{\infty} f(s) d s=\infty$ the solution is global in time $([15])$.

In the following we assume $f$ to satisfy

$$
\begin{gathered}
f(s)>0, \quad f^{\prime}(s)<0, \quad s \geqslant 0, \\
\int_{0}^{\infty} f(s) d s<\infty
\end{gathered}
$$

for instance either $f(s)=e^{-s}$ or $f(s)=(1+s)^{-p}, \quad p>1$, satisfy (1.2). In addition for the initial data it is required that $u_{0}(x), u_{0}^{\prime}(x)$ to be bounded, and that $u_{0}(x) \geqslant 0$ in $[0,1]$ (the last requirement is a consequence of the fact that for any initial data the solution $u$ becomes non-negative over $(0,1]$ for some time $t$ and thus, with an appropriate redefinition of $t$, we can always make this assumption $[13,15])$.

The corresponding steady problem to (1.1) is

$$
w^{\prime}=\lambda \frac{f(w)}{\left(\int_{0}^{1} f(w) d x\right)^{2}}, \quad 0<x<1, \quad w(0)=0,
$$

where $w=w(x)=w(x ; \lambda)$, (see $[6,8,13,14,15])$. For example in the case that $f(s)=e^{-s}$, $w(x)=\ln \left(\lambda x / \mu^{2}+1\right)$ for $\mu>0$ being the root of the equation $\sqrt{\mu} \ln \left(\lambda / \mu^{2}+1\right)=\lambda$.

Under the assumptions (1.2), problem (1.3) has at least one classical (regular) steady solution $([15,17]) w^{*}=w\left(x ; \lambda^{*}\right)$, (more than one $w^{*}$ may exist). In the following, we assume that $w^{*}$ is unique, and that the pair $(\underline{w}, \bar{w})$ at $\lambda<\lambda^{*}$ ( $\lambda$ close to $\left.\lambda^{*}\right)$ has the property: $\underline{w}=w_{1}$ is stable while $\bar{w}=w_{2}$ is unstable, (since without loss of generality it can be required only the existence of at least one $w^{*}$ at $\lambda^{*}$ and that $\underline{w}(x)<\bar{w}(x)$ for $x$ in $(0,1]$ where $\bar{w}$ is the next steady solution greater than $\underline{w}(x)$ at $\left.\lambda<\lambda^{*}\right)$.

It is known that if $(1.2 \mathrm{~b})$ holds, then there exists a critical value of the parameter $\lambda$, which can be identified, amongst other things, with the square (is actually proportional) of the applied potential difference $V$, say $\lambda^{*}<\infty$, such that for $\lambda>\lambda^{*}$, the solution $u(x, t ; \lambda)$ to problem (1.1), blows up globally in finite time $t^{*}\left(u \rightarrow \infty\right.$ for all $x \in(0,1]$ as $t \rightarrow t^{*}-$, [15]) and problem (1.3) has no solutions (of any kind). For a fixed $\lambda \in\left(0, \lambda^{*}\right)$ there exist at least two solutions $w(x ; \lambda)$ and a unique $u(x, t ; \lambda) ; u(x, t ; \lambda)$ may either exist for all time or blow up globally depending on the initial data (for the blow-up, $u_{0}$ must be greater than the greatest steady solution $w(x ; \lambda)$ and $(1.2)$ holds) $[13,14,15]$.

A numerical computation of problem (1.1), by using the upwind scheme has already been presented in [17]. Although it has not appear yet in the bibliography a theoretical analysis of finite difference schemes that can be applied for the numerical solution of nonlocal problems having similar form as problem (1.1). In this work we study initially two explicit finite difference schemes: upwind and Lax -Wendroff, regarding their consistency, stability and convergence. Initially the upwind scheme is applied to problem (1.1) and it is shown that this is first order convergent, if we apply an appropriate discretization to the nonlocal source term. As a next step we apply the Lax - Wendroff scheme in order to get a more accurate numerical approximation. This scheme is of second order accuracy. This accuracy is obtained by the addition of extra correction terms in the discretization of the nonlocal source term. The analysis for both methods holds in the case that $f$ is a decreasing function, if (1.2b) holds for $\lambda<\lambda^{*}$, and for small initial data, or if $\int_{0}^{\infty} f(s) d s=\infty$, so that 
the solution to problem (1.1) exists for all times. In addition it should be mentioned that the results stated in this work are valid, with minor modifications in the proofs, if we also consider an increasing $f$. Moreover, a high resolution method combining the above methods is presented and it is shown that it is total-variation-stable.

An interesting aspect in the study of a numerical solution of such a non-local problem is to investigate the effect of the non-local term in the numerical approximation of the solution. The present analysis for these two methods indicates that other standard methods applied for first order homogeneous hyperbolic equation should result in the same order of convergence for this non-local problem. This can be done if the nonlocal source term is discretized appropriately, if necessary, with the addition of extra correction terms, and the integration method, used to approximate the non-local term, is of the same order. Moreover, as it is apparent from the application of the Lax-Wendroff method to this problem, additional correction terms, related with the derivatives of the non-local source term, must be included in the scheme in order to obtain second order accuracy in both space and time. Similar analysis for other non-local problems exist in the bibliography (e.g [1, 7]). More specifically in [1] an approach based on the method of characteristics is followed. This in principle can be applied also for the solution of problem (1.1). For $s=x+t$ equation (1.1b) becomes $\frac{d u(s)}{d s}=$ $\lambda f(u(s)) /\left[\int_{t}^{1+t} f(u(s)) d s\right]^{2}$. Then this equation can be integrated along the characteristics resulting, e.g in a numerical scheme of the form $U_{j+1}^{n+1}=U_{j}^{n}+h \lambda f\left(U_{j}^{n}\right) / I_{h}^{2}\left(U^{n}\right)$, where $h$ is a time step. The analysis in [1], indicates that in our case, due to the fact that the non local term $\int_{0}^{1} f(u) d x$, is a function of time, extra care should be needed to obtain and analyze a method of higher order accuracy by the method of characteristics. In addition the upwind and the Lax-Wendroff method can be generalized in a more natural way to problems of the form $u_{t}+(g(u))_{x}=\lambda F(u)$. Thus in the present work we will not consider further this method.

Note also that error estimates for a method approximating the solution of the problem in the case of blow-up are important in order to investigate characteristics of the blow-up phenomenon, e.g. the blow-up time, useful for the point of view of applications. Similar works that investigate the convergence of a numerical solution during blow-up have been done, for a parabolic problem, in $[2,3,11]$. In our case, for example, for $f$ being an increasing function a discontinuity in the initial data may cause blow-up of the solution [15]. Aiming to apply a similar approach as in $[2,3]$ for a non-local problem such as problem (1.1), a high resolution method based on a combination of an upwind and a Lax-Wendroff method, which is also introduced and analyzed in this work, would be useful (see [16], Chapter 6).

In section 2 we present the appropriate notations and definitions and we consider the upwind method regarding its consistency, stability and convergence. In section 3 we establish similar results for the Lax-Wendroff method and we introduce a high resolution method which is shown to be TV-stable. Finally in section 4 we present some numerical experiments in support of the results obtained in the previous sections and in section 5 we present the conclusions and some open problems regarding the continuation of this work. 


\section{The Upwind Scheme}

\subsection{Notations}

We introduce a spatial grid $x_{j}=j \Delta x, j=0,1, \ldots, J$, where $\Delta x=1 / J$ is the mesh size and $J$ is a positive integer. We also consider a fixed time interval $0 \leqslant t \leqslant T$. The step length in time is denoted by $\Delta t$ and $t_{n}=n \Delta t, n=0,1,2, \ldots, N$, with $N=[T / \Delta t]$, are the discrete time levels. Also $r=\frac{\Delta t}{\Delta x}$.

We consider the set $H=\{\Delta x>0: \Delta x=1 / J, J \in \mathbb{N}\}$ and for $\Delta x \in H$ we define the vector spaces : $X=Y=\left(\mathbb{R}^{J+1}\right)^{N+1}$. Also if $V=\left(V_{0}, V_{1}, \ldots, V_{J}\right) \in \mathbb{R}^{J+1}$ we define $\|V\|_{\infty}:=\max _{0 \leqslant j \leqslant J}\left|V_{j}\right|$ and $\|V\|_{1}:=\sum_{j=0}^{J^{\prime \prime}} \Delta x\left|V_{j}\right|$, where the " means that the first and last terms of the sum are halved, i.e. the trapezoidal rule is used. For $V=\left(V^{0}, V^{1}, \ldots, V^{N}\right) \in X$, with $V^{n} \in \mathbb{R}^{J+1}$ we define the following norm on $X,\|V\|_{X}:=\max _{0 \leqslant n \leqslant N}\left\|V^{n}\right\|_{1}$. In addition if $V \in Y$ then we define the norm $\|V\|_{Y}:=\left\|V^{0}\right\|_{1}+\sum_{n=1}^{N} \Delta t\left\|V^{n}\right\|_{1}$. Let $R$ be a fixed positive constant and denote by $B\left(u_{h}, R\right)$ the open ball with center $u_{h}$ and radius $R$ of the space $X$ endowed with the norm of $X$ as it is defined above.

For a time step $\Delta t$ and $\Delta x \in H$ we consider the element $u_{h} \in X, u_{h}=\left(u^{0}, u^{1}, \ldots, u^{N}\right) \in$ $X$, with $u^{n}=\left(u_{0}^{n}, u_{1}^{n}, \ldots, u_{J}^{n}\right) \in \mathbb{R}^{J+1}$ and $u_{j}^{n}=u\left(x_{j}, t_{n}\right)$ where $u$ is the exact solution of the problem (1.1). In a similar way we denote by $U_{h} \in X$ the approximate numerical solution of problem (1.1), with $U_{j}^{n}$ being the approximation of the solution at the point $\left(x_{j}, t_{n}\right)$.

Also we use the notations $I\left(u^{n}\right)=\int_{0}^{1} u\left(x, t_{n}\right) d x$ and $F\left(u_{j}^{n}\right)=\frac{f\left(u_{j}^{n}\right)}{I^{2}\left(u^{n}\right)}$. By $I_{h}$ we denote the numerical approximation of $I$, i.e. $I_{h}\left(u^{n}\right)=\sum_{j=0}^{J^{\prime \prime}} \Delta x u_{j}^{n}$. In this case we have

$$
\left|I_{h}\left(u^{n}\right)-I\left(u^{n}\right)\right|=O\left(\Delta x^{2}\right) .
$$

Finally we set $F_{h}\left(u_{j}^{n}\right)=\frac{f\left(u_{j}^{n}\right)}{I_{h}^{2}\left(u^{n}\right)}$.

Note that $C, c, c_{i}, M_{i}, i=1,2, \ldots$ will denote positive constants independent of $\Delta x, \Delta t, n$ $(0 \leqslant n \leqslant N)$ and $j,(0 \leqslant j \leqslant J)$ having possibly different values at different places.

\subsection{Formulation and Analysis of the Numerical Method}

An upwind scheme applied to problem (1.1) gives:

$$
\begin{aligned}
& U_{0}^{n+1}=0, \\
& \quad U_{j}^{n+1}=U_{j}^{n}-r\left(U_{j}^{n}-U_{j-1}^{n}\right)+\lambda \Delta t F_{h}\left(U_{j}^{n}\right), \quad j=1, \ldots, J,
\end{aligned}
$$

for $0 \leqslant n \leqslant N-1$ and with $U^{0}=\left(U_{0}^{0}, U_{1}^{0}, \ldots, U_{J}^{0}\right)$ known.

Next we introduce the mapping $\phi_{h}: B\left(u_{h}, R\right) \subset X \rightarrow Y$ defined by the equations

$$
\begin{aligned}
& \phi_{h}\left(V^{0}, V^{1}, \ldots, V^{N}\right)=\left(Z^{0}, Z^{1}, \ldots, Z^{N}\right), \\
& Z^{0}=V^{0}-U^{0}, \\
& Z_{0}^{n+1}=0, \\
& Z_{j}^{n+1}=\frac{1}{\Delta t}\left(V_{j}^{n}-\frac{\Delta t}{\Delta x}\left(V_{j}^{n}-V_{j-1}^{n}\right)+\Delta t \lambda \frac{f\left(V_{j}^{n}\right)}{I_{h}^{2}\left(V^{n}\right)}-V_{j}^{n+1}\right) \quad 1 \leqslant j \leqslant J,
\end{aligned}
$$

for $0 \leqslant n \leqslant N-1$. Then $U_{h}=\left(U^{0}, U^{1}, \ldots, U^{N}\right)$ is a solution of the scheme (2.1) if and only if $\phi_{h}\left(U_{h}\right)=\left(R^{0}, R^{1}, \ldots, R^{N}\right)$, with $R^{n}=0 \in \mathbb{R}^{J+1}, 0 \leqslant n \leqslant N$.

In the following we study the consistency, the stability and the convergence of the scheme $(2.1)$. 


\section{Consistency}

We define the local descretization error as $l_{h}=\phi_{h}\left(u_{h}\right) \in Y$ and we say that the descritization is consistent if as $\Delta x, \Delta t \rightarrow 0, \lim _{\Delta x, \Delta t \rightarrow 0}\left\|\phi_{h}\left(u_{h}\right)\right\|_{Y}=\lim _{\Delta x, \Delta t \rightarrow 0}\left\|l_{h}\right\|_{Y}=0$.

Proposition 1. Assuming that $f$ satisfies condition (1.2a) and $u$ is $C^{2}$ global bounded solution of problem (1.1) (i.e. the initial data are smooth enough and $\lambda \leqslant \lambda^{*}, u_{0}(x)<w_{2}(x)$ if (1.2b) holds or $\int_{0}^{\infty} d s / f(s)=\infty$ ) then if for $u_{0}\left(x_{j}\right)=u_{j}^{0}, j=0,1, \ldots, J$, we have $\left\|u^{0}-U^{0}\right\|_{1}=o(1)$ the local discretization error satisfies the condition

$$
\left\|\phi_{h}\left(u_{h}\right)\right\|_{Y}=O(\Delta t+\Delta x) .
$$

Proof. We denote $\phi_{h}\left(u_{h}\right)=\left(u^{0}-U^{0}, \tau^{1}, \tau^{2}, \ldots, \tau^{N}\right)$, where $\tau^{n}, 1 \leqslant n \leqslant N$ are the local truncation errors to be bounded. Indeed for $j=1, \ldots, J$ we have

$$
\begin{aligned}
\left|\tau_{j}^{n+1}\right|= & \frac{1}{\Delta t}\left|u_{j}^{n}-\frac{\Delta t}{\Delta x}\left(u_{j}^{n}-u_{j-1}^{n}\right)+\Delta t \lambda F_{h}\left(u_{j}^{n}\right)-u_{j}^{n+1}\right| \\
= & \frac{1}{\Delta t} \mid u_{j}^{n}-\frac{\Delta t}{\Delta x}\left(u_{j}^{n}-u_{j}^{n}+\Delta x u_{x_{j}}^{n}-\frac{1}{2} \Delta x^{2} u_{x x j}^{n}+\cdots\right) \\
& \quad+\Delta t \lambda F_{h}\left(u_{j}^{n}\right)-u_{j}^{n}-\Delta t u_{t j}^{n}-\frac{1}{2} \Delta t^{2} u_{t t j}^{n}+\cdots \mid \\
= & \left|-u_{x_{j}}^{n}-u_{t j}^{n}+\lambda F_{h}\left(u_{j}^{n}\right)-\frac{1}{2} \Delta t u_{t t j}^{n}-\frac{1}{2} \Delta x u_{x x j}^{n}\right|+\cdots \\
\text { or }\left|\tau_{j}^{n+1}\right| \leqslant & \frac{1}{2} \Delta t\left|u_{t t_{j}^{n}}^{n}\right|+\frac{1}{2} \Delta x\left|u_{x x_{j}}^{n}\right|+\lambda f\left(u_{j}^{n}\right)\left|\frac{1}{I_{h}^{2}\left(u^{n}\right)}-\frac{1}{I^{2}\left(u^{n}\right)}\right| .
\end{aligned}
$$

Regarding the third term in equation (2.2) we have that

$$
\lambda f\left(u_{j}^{n}\right)\left|\frac{1}{I_{h}^{2}\left(u^{n}\right)}-\frac{1}{I^{2}\left(u^{n}\right)}\right|=\lambda f\left(u_{j}^{n}\right) \frac{\left[I_{h}\left(u^{n}\right)+I\left(u^{n}\right)\right]}{I_{h}^{2}\left(u^{n}\right) I^{2}\left(u^{n}\right)}\left|I_{h}\left(u^{n}\right)-I\left(u^{n}\right)\right| .
$$

Since $u$ is bounded in $[0,1] \times[0, T]$ there exists a constant $M_{u}$ such that $u\left(x, t_{n}\right) \leqslant M_{u}$, $0 \leqslant n \leqslant N$. Therefore $f\left(u^{n}\right) \geqslant f\left(M_{u}\right)>0$ and $\frac{1}{I_{h}^{2}\left(u^{n}\right)}=\frac{1}{\left[\sum_{j=0}^{J^{\prime \prime}} \Delta x f\left(u_{j}^{n}\right)\right]^{2}} \leqslant \frac{1}{f\left(M_{u}\right)^{2}}$. Similarly $\frac{1}{I^{2}\left(u^{n}\right)}=\frac{1}{\left[\int_{0}^{1} f\left(u^{n}\right) d x\right]^{2}} \leqslant \frac{1}{f\left(M_{u}\right)^{2}}$. Also for $f$ decreasing and $f(0)>0$ we have, $f\left(u_{j}^{n}\right) \leqslant f(0)$, $I_{h}\left(u^{n}\right)=\sum_{j=1}^{J^{\prime \prime}} \Delta x f\left(u_{j}^{n}\right) \leqslant f(0)$ and $I\left(u^{n}\right)=\int_{0}^{1} f\left(u^{n}\right) d x \leqslant f(0)$. Therefore due to the fact that $\left|I_{h}\left(u^{n}\right)-I\left(u^{n}\right)\right|=O\left(\Delta x^{2}\right)$ we deduce that

$$
\lambda f\left(u_{j}^{n}\right)\left|\frac{1}{I_{h}^{2}\left(u^{n}\right)}-\frac{1}{I\left(u^{n}\right)^{2}}\right| \leqslant \frac{2 \lambda f(0)^{2}}{f\left(M_{u}\right)^{4}}\left|I_{h}\left(u^{n}\right)-I\left(u^{n}\right)\right|=O\left(\Delta x^{2}\right) .
$$

We have $\tau_{0}^{n}=0$, for $1 \leqslant n \leqslant N$. Therefore combining also equations (2.2) and (2.3) we have that

$$
\left|\tau_{j}^{n}\right|=O(\Delta t+\Delta x), \quad 0 \leqslant j \leqslant J, \quad 1 \leqslant n \leqslant N,
$$

and hence, using the assumption that $\left\|u^{0}-U^{0}\right\|_{1}=o(1)$, we deduce that $\lim _{\Delta x, \Delta t \rightarrow 0}\left\|\phi_{h}\left(u_{h}\right)\right\|_{Y}$ $=\lim _{\Delta x, \Delta t \rightarrow 0}|| l_{h} \|_{Y}=0$ and that the scheme is consistent.

Remark The previous proposition can be easily modified to include also the case that $f$ is an increasing function. Indeed if $f(s)>0, f^{\prime}(s)>0$, for $s \geqslant 0$ and problem (1.1) attains a $C^{2}$ bounded global in time solution, $u(x, t)$, then we have $0<f(0)<f(u(x, t))<f\left(M_{u}\right)$, where 
$M_{u}$ is an upper bound of $u$ for $0 \leqslant x \leqslant 1$ and $t>0$. Therefore $\frac{1}{I_{h}^{2}\left(u^{n}\right)}=\frac{1}{\left[\sum_{j=0}^{J^{\prime \prime}} \Delta x f\left(u_{j}^{n}\right)\right]^{2}} \leqslant \frac{1}{f(0)^{2}}$ and $\frac{1}{I^{2}\left(u^{n}\right)}=\frac{1}{\left[\int_{0}^{1} f\left(u^{n}\right) d x\right]^{2}} \leqslant \frac{1}{f(0)^{2}}$. Also, $f\left(u_{j}^{n}\right) \leqslant f\left(M_{u}\right), I_{h}\left(u^{n}\right)=\sum_{j=1}^{J^{\prime \prime}} \Delta x f\left(u_{j}^{n}\right) \leqslant f\left(M_{u}\right)$ and $I\left(u^{n}\right)=\int_{0}^{1} f\left(u^{n}\right) d x \leqslant f\left(M_{u}\right)$. Hence

$$
\lambda f\left(u_{j}^{n}\right)\left|\frac{1}{I_{h}^{2}\left(u^{n}\right)}-\frac{1}{I\left(u^{n}\right)^{2}}\right| \leqslant \frac{2 \lambda f\left(M_{u}\right)^{2}}{f(0)^{4}}\left|I_{h}\left(u^{n}\right)-I\left(u^{n}\right)\right|=O\left(\Delta x^{2}\right) .
$$

Likewise the rest of the propositions in this work can be modified in a similar manner to include the case where $f$ is increasing.

\section{Stability}

In the following we show that the scheme is stable. For each $\Delta x$ and $\Delta t$ let $M_{h}>0$ a constant. We say that the discretization (2.1) is stable for $u_{h}$ restricted to the thresholds $M_{h}$ if there exist two positive constants $r_{0}$ and $S$ such that for $r=\frac{\Delta t}{\Delta x} \leqslant r_{0}, B\left(u_{h}, M_{h}\right)$ is contained in the domain of $\phi_{h}$ and for every $V, W \in B\left(u_{h}, M_{h}\right),\|V-W\|_{X} \leqslant S\left\|\phi_{h}(V)-\phi_{h}(W)\right\|_{Y}$.

Proposition 2. Under the hypotheses of proposition (1) the discretization (2.1) is stable for $r=\frac{\Delta t}{\Delta x} \leqslant 1$.

Proof. Let $V, W \in B\left(u_{h}, M_{h}\right)$ of $X$ with $\phi_{h}(V)=Z$ and $\phi_{h}(W)=S$. We set $E^{n}=$ $V^{n}-W^{n} \in \mathbb{R}^{J+1}, 0 \leqslant n \leqslant N$. We have $E_{0}^{n}=0$, for $1 \leqslant n \leqslant N$ and for $0 \leqslant n \leqslant N-1$, $1 \leqslant j \leqslant J$, that

$$
\begin{aligned}
\left|E_{j}^{n+1}\right|= & \left|V_{j}^{n+1}-W_{j}^{n+1}\right| \\
= & \mid V_{j}^{n}-\frac{\Delta t}{\Delta x}\left(V_{j}^{n}-V_{j-1}^{n}\right)+\Delta t \lambda F_{h}\left(V_{j}^{n}\right) \\
& -W_{j}^{n}+\frac{\Delta t}{\Delta x}\left(W_{j}^{n}-W_{j-1}^{n}\right)-\Delta t \lambda F_{h}\left(W_{j}^{n}\right)-\Delta t\left(Z_{j}^{n+1}-S_{j}^{n+1}\right) \mid \\
\leqslant & (1-r)\left|V_{j}^{n}-W_{j}^{n}\right|+r\left|V_{j-1}^{n}-W_{j-1}^{n}\right|+\lambda \Delta t\left|F_{h}\left(V_{j}^{n}\right)-F_{h}\left(W_{j}^{n}\right)\right| \\
& -\Delta t\left|Z_{j}^{n+1}-S_{j}^{n+1}\right| .
\end{aligned}
$$

By the assumptions on $f$ we have that $f$ is locally Lipschitz, i.e. $\left|f\left(V_{j}^{n}\right)-f\left(W_{j}^{n}\right)\right| \leqslant$ $L\left|V_{j}^{n}-W_{j}^{n}\right|$ for a constant $L>0$. In addition $0<f\left(V_{j}^{n}\right) \leqslant f(0)$ for $f$ decreasing and for $V, W \in B\left(u_{h}, M_{h}\right)$ we have that $\frac{1}{I_{h}\left(V^{n}\right)^{2}} \leqslant \frac{1}{f\left(M_{h}\right)^{2}}, \frac{1}{I_{h}\left(W^{n}\right)^{2}} \leqslant \frac{1}{f\left(M_{h}\right)^{2}}$ and $I_{h}\left(V^{n}\right) \leqslant f(0)$, $I_{h}\left(W^{n}\right) \leqslant f(0)$. Also $\left|I_{h}\left(V^{n}\right)-I_{h}\left(W^{n}\right)\right| \leqslant \sum_{j=0}^{J^{\prime \prime}} \Delta x\left|f\left(V_{j}^{n}\right)-f\left(W_{j}^{n}\right)\right| \leqslant L \sum_{j=0}^{J^{\prime \prime}} \Delta x \mid V_{j}^{n}-$ $W_{j}^{n} \mid=L\left\|E^{n}\right\|_{1}$. Thus

$$
\begin{aligned}
\left|F_{h}\left(V_{j}^{n}\right)-F_{h}\left(W_{j}^{n}\right)\right| & =\left|\frac{f\left(V_{j}^{n}\right)}{I_{h}\left(V^{n}\right)}-\frac{f\left(W_{j}^{n}\right)}{I_{h}\left(W^{n}\right)}\right| \\
& \leqslant\left|\frac{f\left(V_{j}^{n}\right)}{I_{h}\left(V^{n}\right)}-\frac{f\left(V_{j}^{n}\right)}{I_{h}\left(W^{n}\right)}\right|+\left|\frac{f\left(V_{j}^{n}\right)}{I_{h}\left(W^{n}\right)}-\frac{f\left(W_{j}^{n}\right)}{I_{h}\left(W^{n}\right)}\right| \\
& \leqslant\left|f\left(V_{j}^{n}\right)\right| \frac{\left|I_{h}\left(V^{n}\right)+I_{h}\left(W^{n}\right)\right|}{I_{h}^{2}\left(V^{n}\right) I_{h}^{2}\left(W^{n}\right)}\left|I_{h}\left(V^{n}\right)-I_{h}\left(W^{n}\right)\right| \\
& \quad+\frac{1}{I_{h}^{2}\left(W^{n}\right)}\left|f\left(V_{j}^{n}\right)-f\left(W_{j}^{n}\right)\right| \\
\leqslant & \frac{2 f^{2}(0)}{f^{4}\left(M_{h}\right)}\left|I_{h}\left(V^{n}\right)-I_{h}\left(W^{n}\right)\right|+\frac{L}{f^{2}\left(M_{h}\right)}\left|V_{j}^{n}-W_{j}^{n}\right| .
\end{aligned}
$$


Therefore for $0<r<1, c_{1}=\frac{\lambda L}{f^{2}\left(M_{h}\right)}$ and $c_{2}=\frac{2 \lambda L f^{2}(0)}{f^{4}\left(M_{h}\right)}$ we obtain

$$
\left|E_{j}^{n+1}\right| \leqslant\left[(1-r)+c_{1} \Delta t\right]\left|E_{j}^{n}\right|+r\left|E_{j-1}^{n}\right|+c_{2}|| E^{n} \|_{1}+\Delta t\left|Z_{j}^{n+1}-S_{j}^{n+1}\right| .
$$

For $c=\max \left(c_{1}, c_{2}\right)$ we deduce that

$$
\begin{aligned}
\left\|E^{n+1}\right\|_{1} & \leqslant[(1-r)+c \Delta t]\left\|E^{n}\right\|_{1}+r\left\|E^{n}\right\|_{1}+\Delta t\left\|Z_{j}^{n+1}-S_{j}^{n+1}\right\|_{1} \\
& \leqslant(1+c \Delta t)\left\|E^{n}\right\|_{1}+\Delta t\left\|Z^{n+1}-S^{n+1}\right\|_{1} .
\end{aligned}
$$

Applying the above relation recursively we have

$$
\left\|E^{n+1}\right\|_{1} \leqslant C\left(\left\|E^{0}\right\|_{1}+\Delta t \sum_{m=1}^{n+1}\left\|S^{m}-Z^{m}\right\|_{1}\right),
$$

for some constant $C$. Therefore by the discrete Gronwall lemma we get

$$
\max _{0 \leqslant n \leqslant N}\left\|E^{n}\right\|_{1} \leqslant C\left(\left\|E^{0}\right\|_{1}+\Delta t \sum_{n=1}^{N}\left\|S^{n}-Z^{n}\right\|_{1}\right)=C\left\|\phi_{h}(V)-\phi_{h}(W)\right\|_{Y}
$$

and thus $\|V-W\|_{X} \leqslant C\left\|\phi_{h}(V)-\phi_{h}(W)\right\|_{Y}$.

\section{Convergence}

Regarding the convergence of the scheme we have the following proposition:

Proposition 3. Assuming that the hypotheses of proposition (1) hold and that $U^{0}$ is such that $\left\|u^{0}-U^{0}\right\|_{1}=O(\Delta x)$, as $\Delta x \rightarrow 0$ then the numerical solution of the scheme $U_{h}$ satisfies

$$
\left\|U_{h}-u_{h}\right\|_{X}=O(\Delta t+\Delta x)
$$

and

$$
\left\|U_{h}-u_{h}\right\|_{\infty}=O(\Delta t+\Delta x)
$$

as $\Delta x, \Delta t \rightarrow 0$.

Proof. We have that $\phi_{h}$ is continuous and stable on $B\left(u_{h}, M_{h}\right)$. Hence (see [21], [1]) there exist the inverse $\phi_{h}^{-1}$ defined on $B\left(u_{h}, M_{h} / S\right)$ for $S$ being the stability constant. We consider the vector $R=\left(R^{0}, R^{1}, \ldots, R^{N}\right) \in X$ such that $\phi_{h}\left(U_{h}\right)=R$ with $R^{n}=0 \in \mathbb{R}^{J+1}$, $0 \leqslant n \leqslant N$. Then $U_{h}$ exists and is the unique solution of the scheme.

By the consistency property and by the fact that $\left\|U^{0}-u^{0}\right\|_{1}=O(\Delta x)$ we have that $\left\|\phi_{h}\left(u_{h}\right)-R\right\|_{Y}=\left\|\phi_{h}\left(u_{h}\right)\right\|_{Y}=O(\Delta t+\Delta x)$. Thus for $\Delta x, \Delta t$ small enough $U_{h} \in B\left(u_{h}, M_{h}\right)$ and by the stability property, i.e. the relation $\left\|U_{h}-u_{h}\right\|_{X} \leqslant C\left\|\phi_{h}\left(u_{h}\right)-\phi_{h}\left(U_{h}\right)\right\|_{Y}$, we have that

$$
\left\|U_{h}-u_{h}\right\|_{X} \leqslant C\left\|\phi_{h}\left(u_{h}\right)-\phi_{h}\left(U_{h}\right)\right\|_{Y}=C\left\|\phi_{h}\left(u_{h}\right)-R\right\|_{Y}=O(\Delta t+\Delta x) .
$$

It remains to prove that $\left\|U_{h}-u_{h}\right\|_{\infty}=O(\Delta t+\Delta x)$.

We set $e^{n}:=U^{n}-u^{n}$ and $L\left(V_{j}^{n}\right):=V_{j}^{n}-\frac{\Delta t}{\Delta x}\left(V_{j}^{n}-V_{j-1}^{n}\right)+\Delta t \lambda F\left(V_{j}^{n}\right)$. Then $e_{0}^{n}=0$ for $1 \leqslant n \leqslant N$ and for $1 \leqslant j \leqslant J$ we have

$$
\begin{aligned}
e_{j}^{n+1} & =U_{j}^{n+1}-u_{j}^{n+1}=L\left(U_{j}^{n}\right)-u_{j}^{n+1}=\left(L\left(U_{j}^{n}\right)-L\left(u_{j}^{n}\right)\right)+\left(L\left(u_{j}^{n}\right)-u_{j}^{n+1}\right) \\
& =L\left(U_{j}^{n}\right)-L\left(u_{j}^{n}\right)+\Delta t \tau_{j}^{n+1},
\end{aligned}
$$


where $\left|\tau_{j}^{n+1}\right|=O(\Delta t+\Delta x)$. Also

$$
\left|L\left(U_{j}^{n}\right)-L\left(u_{j}^{n}\right)\right| \leqslant(1-r)\left|e_{j}^{n}\right|+r\left|e_{j-1}^{n}\right|+\lambda \Delta t\left|F_{h}\left(U_{j}^{n}\right)-F_{h}\left(u_{j}^{n}\right)\right|,
$$

and for $\Delta x, \Delta t$ small enough $U_{h} \in B\left(u_{h}, M_{h}\right)$, so as it is stated in relation (2.4) we have $\left|F_{h}\left(U_{j}^{n}\right)-F_{h}\left(u_{j}^{n}\right)\right| \leqslant c_{1}\left|e_{j}^{n}\right|+c_{2}|| e^{n} \|_{1}$. Therefore

$$
\left|L\left(V_{j}^{n}\right)-L\left(W_{j}^{n}\right)\right| \leqslant(1+c \Delta t)\left\|e^{n}\right\|_{1},
$$

and

$$
\left|e_{j}^{n+1}\right| \leqslant(1+c \Delta t)\left\|e^{n}\right\|_{1}+\Delta t\left|\tau_{j}^{n+1}\right|, \quad j=1, \ldots, J .
$$

Thus

$$
\left\|e^{n}\right\|_{\infty}=\max _{0 \leqslant j \leqslant J}\left|e^{n}\right| \leqslant(1+c \Delta t)\left\|e^{n}\right\|_{1}+O(\Delta t+\Delta x)
$$

and in addition from equation (2.5) we have that

$$
\left\|e^{n+1}\right\|_{1} \leqslant(1+c \Delta t)\left\|e^{n}\right\|_{1}+O(\Delta t+\Delta x) .
$$

Therefore by the relations (2.6) and (2.7) recursively we obtain

$$
\left\|e^{n}\right\|_{\infty} \leqslant C\left\|e^{0}\right\|_{1}+O(\Delta t+\Delta x)
$$

for some constant $C$ and for every $n, 1 \leqslant n \leqslant N$. Finally we deduce that

$$
\max _{0 \leqslant n \leqslant N}\left\|e^{n}\right\|_{\infty}=\max _{0 \leqslant n \leqslant N}\left\|U^{n}-u^{n}\right\|_{\infty} \leqslant C\left\|e^{0}\right\|_{1}+O(\Delta t+\Delta x),
$$

and taking also into account that $\left\|e^{0}\right\|_{1}=\left\|U^{0}-u^{0}\right\|_{1}=O(\Delta x)$ we get the required result

$$
\max _{0 \leqslant n \leqslant N}\left\|e^{n}\right\|_{\infty}=\max _{0 \leqslant n \leqslant N}\left\|U^{n}-u^{n}\right\|_{\infty}=O(\Delta t+\Delta x) .
$$

\section{The Lax - Wendroff Scheme}

For the following analysis we will use the notations stated in the previous section. Also

for convenience we will denote by $I_{1}(u):=\int_{0}^{1} f^{\prime}(u) f(u) d x$ and accordingly $I_{1 h}\left(U^{n}\right):=$ $\sum_{j=0}^{J^{\prime \prime}} f^{\prime}\left(U_{j}^{n}\right) f\left(U_{j}^{n}\right)$. In the following $f^{\prime}$ is assumed to be locally Lipschitz, with constant $L^{\prime}$ and bounded i.e. $\left|f^{\prime}(s)\right| \leqslant M_{1}$ for $s \geqslant 0$ and some constant $M_{1}$.

In order to derive a Lax - Wendroff Scheme for problem (1.1) we note that $u_{t}=-u_{x}+$ $\lambda F(u)$ and $u_{t t}=u_{x x}+\lambda\left(F_{t}(u)-F_{x}(u)\right)$. Thus by expanding $u(x, t+\Delta t)$ about the point $(x, t)$ we obtain

$$
\begin{aligned}
u(x, t+\Delta t)=u(x, t) & -\Delta t u_{x}(x, t)+\frac{\Delta t^{2}}{2} u_{x x}(x, t)+\lambda \Delta t F(u(x, t)) \\
& +\lambda \frac{\Delta t^{2}}{2}\left(F_{t}(u(x, t))-F_{x}(u(x, t))\right)+O\left(\Delta t^{3}\right) .
\end{aligned}
$$


Note also that by using the fact that $u_{t}=-u_{x}+\lambda F(u)$ we have $G(u):=F_{t}(u)-F_{x}(u)=$ $G_{1}(u)+G_{2}(u) u_{x}+G_{3}(u)$ where the expressions for $G_{i}, i=1,2,3$ are $G_{1}(u)=\lambda \frac{f^{\prime}(u) f(u)}{I^{4}(u)}$, $G_{2}(u)=-2 \frac{f^{\prime}(u)}{I^{2}(u)}$, and $G_{3}(u)=-2 \frac{f(u)}{I^{3}(u)}\left(\lambda \frac{I_{1}(u)}{\left.I^{2}(u)\right)}-(f(u(1, t))-f(u(0, t)))\right.$. By taking central differences for the approximation of $u_{x}$ and $u_{x x}$ we can derive the following Lax - Wendroff scheme

$$
\begin{aligned}
& U_{0}^{n+1}=0, \\
& U_{j}^{n+1}=\frac{r}{2}(1+r) U_{j-1}^{n}+\left(1-r^{2}\right) U_{j}^{n}-\frac{r}{2}(1-r) U_{j+1}^{n}+\lambda \Delta t F_{h}\left(U_{j}^{n}\right)+\lambda \frac{\Delta t^{2}}{2} G_{h}\left(U_{j}^{n}\right), \\
& U_{J}^{n+1}=\left(1-\frac{3 r}{2}+\frac{r^{2}}{2}\right) U_{J}^{n}+\left(2 r-r^{2}\right) U_{J-1}^{n}-\frac{r}{2}(1-r) U_{J-2}^{n}+\lambda \Delta t F_{h}\left(U_{J}^{n}\right)+\lambda \frac{\Delta t^{2}}{2} G_{h}\left(U_{J}^{n}\right),
\end{aligned}
$$

for $j=1, \ldots, J-1,0 \leqslant n \leqslant N-1$ and $U^{0}$ known. Also

$$
\begin{aligned}
& G_{h}\left(U_{j}^{n}\right)=G_{1 h}\left(U_{j}^{n}\right)+G_{2 h}\left(U_{j}^{n}\right)\left(\frac{U_{j+1}^{n}-U_{j-1}^{n}}{2 \Delta x}\right)+G_{3 h}\left(U_{j}^{n}\right), j=1, \ldots, J-1, \\
& G_{h}\left(U_{J}^{n}\right)=G_{1 h}\left(U_{J}^{n}\right)+G_{2 h}\left(U_{J}^{n}\right)\left(\frac{3 U_{J}^{n}-4 U_{J-1}^{n}+U_{J-2}^{n}}{2 \Delta x}\right)+G_{3 h}\left(U_{J}^{n}\right),
\end{aligned}
$$

Where

$$
\begin{gathered}
G_{1 h}\left(U_{j}^{n}\right)=\lambda \frac{f^{\prime}\left(U_{j}^{n}\right) f\left(U_{j}^{n}\right)}{I_{h}^{4}\left(U^{n}\right)}, \quad G_{2 h}\left(U_{j}^{n}\right)=-2 \frac{f^{\prime}\left(U_{j}^{n}\right)}{I_{h}^{2}\left(U_{j}^{n}\right)}, \\
G_{3 h}\left(U_{j}^{n}\right)=-2 \frac{f\left(U_{j}^{n}\right)}{I_{h}^{3}\left(U_{j}^{n}\right)}\left(\lambda \frac{I_{1 h}\left(U^{n}\right)}{\left.I_{h}^{2}\left(U^{n}\right)\right)}-\left(f\left(U_{J}^{n}\right)-f\left(U_{0}^{n}\right)\right) .\right.
\end{gathered}
$$

Note that for the approximation of the solution at the $J$ th point is used one sided, second order approximations, i.e. the Beam Warming method, of the derivatives $u_{x J}^{n}$ and $u_{x x J}^{n}$, in order to maintain the $O\left(\Delta x^{2}\right)$ accuracy of the scheme.

This scheme can give a more accurate approximation of the solution of order $O\left(\Delta t^{2}+\right.$ $\Delta x^{2}$ ), subject to the fact that for the integral of the source term a second order approximation rule is used.

\section{Consistency}

In the present case we redefine the mapping $\phi_{h}: B\left(u_{h}, R\right) \subset X \rightarrow Y$ in the appropriate, for the Lax-Wendroff scheme, way

$$
\begin{aligned}
& \phi_{h}\left(V^{0}, V^{1}, \ldots, V^{N}\right)=\left(Z^{0}, Z^{1}, \ldots, Z^{N}\right), \\
& Z^{0}=V^{0}-U^{0} \text {, } \\
& Z_{0}^{n+1}=0 \text {, } \\
& Z_{j}^{n+1}=\frac{1}{\Delta t}\left(\frac{r}{2}(1+r) V_{j-1}^{n}+\left(1-r^{2}\right) V_{j}^{n}-\frac{r}{2}(1-r) V_{j+1}^{n}+\lambda \Delta t F_{h}\left(V_{j}^{n}\right)\right. \\
& \left.+\lambda \frac{\Delta t^{2}}{2} G_{h}\left(V_{j}^{n}\right)-V_{j}^{n+1}\right), \\
& Z_{J}^{n+1}=\frac{1}{\Delta t}\left(\left(1-\frac{3 r}{2}+\frac{r^{2}}{2}\right) V_{J}^{n}+\left(2 r-r^{2}\right) V_{J-1}^{n}-\frac{r}{2}(1-r) V_{J-2}^{n}+\lambda \Delta t F_{h}\left(V_{J}^{n}\right)\right. \\
& \left.+\lambda \frac{\Delta t^{2}}{2} G_{h}\left(V_{J}^{n}\right)-V_{J}^{n+1}\right),
\end{aligned}
$$

for $j=1, \ldots, J-1$ and $0 \leqslant n \leqslant N-1$. 
Proposition 4. Assuming that $f$ satisfies (1.2a), with $f^{\prime}$ being locally Lipschitz, and u is $C^{3}$ global bounded solution of problem (1.1) (i.e. the initial data are smooth enough and $\lambda \leqslant \lambda^{*}, u_{0}(x)<w_{2}(x)$ if (1.2b) holds or $\left.\int_{0}^{\infty} d s / f(s)=\infty\right)$ then if $\left\|u_{0}(x)-U^{0}\right\|_{1}=o(1)$ the local discretization error for the scheme (3.1b-3.1c) satisfies the relation

$$
\left\|\phi_{h}\left(u_{h}\right)\right\|_{Y}=O\left(\Delta t^{2}+\Delta x^{2}\right) .
$$

Proof. We have $\tau_{0}^{n}=0$, for $1 \leqslant n \leqslant N$ and for $0 \leqslant n \leqslant N-1,1 \leqslant j \leqslant J-1$ that

$$
\begin{aligned}
\left|\tau_{j}^{n+1}\right|=\frac{1}{\Delta t} \mid \frac{r}{2}(1+r) u_{j-1}^{n}+\left(1-r^{2}\right) u_{j}^{n}-\frac{r}{2}(1 & -r) u_{j+1}^{n}+\lambda \Delta t F_{h}\left(u_{j}^{n}\right) \\
& +\lambda \frac{\Delta t^{2}}{2} G_{h}\left(u_{j}^{n}\right)-u_{j}^{n+1} \mid .
\end{aligned}
$$

Therefore

$$
\begin{aligned}
\left|\tau_{j}^{n+1}\right|=\frac{1}{\Delta t} \mid & \frac{r}{2}(1+r)\left(u_{j}^{n}-\Delta x u_{x_{j}}^{n}+\frac{\Delta x^{2}}{2} u_{x x_{j}}^{n}-\frac{\Delta x^{3}}{6} u_{x x x_{j}}{ }^{n}+\cdots\right) \\
+ & \left(1-r^{2}\right) u_{j}^{n} \\
- & \frac{r}{2}(1-r)\left(u_{j}^{n}+\Delta x u_{x_{j}}^{n}+\frac{\Delta x^{2}}{2} u_{x x_{j}}^{n}+\frac{\Delta x^{3}}{6} u_{x x x_{j}}{ }^{n}+\cdots\right) \\
+ & \lambda \Delta t F_{h}\left(u_{j}^{n}\right)+\lambda \frac{\Delta t^{2}}{2} G_{h}\left(u_{j}^{n}\right) \\
& -\left(u_{j}^{n}+\Delta t u_{t j}^{n}+\frac{\Delta t^{2}}{2} u_{t t j}^{n}+\frac{\Delta t^{3}}{6} u_{t t t j}^{n}+\cdots\right) \mid .
\end{aligned}
$$

or

$$
\begin{aligned}
&\left|\tau_{j}^{n+1}\right|=\frac{1}{\Delta t} \mid-\Delta t\left(u_{t_{j}}^{n}+u_{x_{j}}^{n}\right)+\lambda \Delta t F_{h}\left(u_{j}^{n}\right)+\frac{\Delta t^{2}}{2}\left(u_{x x_{j}^{n}}^{n}-u_{t t j}^{n}+\lambda G_{h}\left(u_{j}^{n}\right)\right) \\
&-\frac{\Delta t \Delta x^{2}}{6} u_{x x x_{j}}^{n}+\frac{\Delta t^{3}}{6} u_{t t t_{j}}^{n}+\cdots \mid \\
&\left.\leqslant\left|\lambda\left(F_{h}\left(u_{j}^{n}\right)-F\left(u_{j}^{n}\right)\right)\right|+\frac{\Delta t}{2}\left(\mid u_{x x_{j}}^{n}-u_{t t_{j}}^{n}+\lambda G_{h}\left(u_{j}^{n}\right)\right) \mid\right) \\
&+\frac{\Delta x^{2}}{6}\left|u_{x x x_{j}^{n}}\right|+\frac{\Delta t^{2}}{6}\left|u_{t t t j}^{n}\right|+\cdots
\end{aligned}
$$

As it is shown in Proposition 1 we have that $\left|F_{h}\left(u_{j}^{n}\right)-F\left(u_{j}^{n}\right)\right|=O\left(\Delta x^{2}\right)$. Also $u_{t t}=$ $u_{x x}+\lambda\left(F_{t}(u)-F_{x}(u)\right)=u_{x x}+\lambda G(u)$, and $u_{x x_{j}}^{n}-u_{t t}^{n}+\lambda G_{h}\left(u_{j}^{n}\right)=\lambda G\left(u_{j}^{n}\right)-\lambda G_{h}\left(u_{j}^{n}\right)$ and in a similar way we have

$$
\left|G_{1}\left(u_{j}^{n}\right)-G_{1 h}\left(u_{j}^{n}\right)\right|=\lambda\left|\frac{f^{\prime}\left(u_{j}^{n}\right) f\left(u_{j}^{n}\right)}{I^{4}\left(u^{n}\right)}-\frac{f^{\prime}\left(u_{j}^{n}\right) f\left(u_{j}^{n}\right)}{I_{h}^{4}\left(u^{n}\right)}\right| \leqslant c_{1}\left|\frac{1}{I^{4}\left(u^{n}\right)}-\frac{1}{I_{h}^{4}\left(u^{n}\right)}\right| \leqslant O\left(\Delta x^{2}\right),
$$

for some constant $c_{1}=\frac{4 \lambda f^{4}(0) M_{1}}{f^{8}\left(M_{u}\right)}$. Also

$$
\begin{aligned}
\left|G_{2}\left(u_{j}^{n}\right) u_{x_{j}}^{n}-G_{2 h}\left(u_{j}^{n}\right) \frac{u_{j+1}^{n}-u_{j-1}^{n}}{2 \Delta x}\right| & =\left|G_{2}\left(u_{j}^{n}\right) u_{x_{j}}^{n}-G_{2 h}\left(u_{j}^{n}\right)\left(u_{x_{j}}^{n}+\frac{\Delta x^{2}}{6} u_{x x x_{j}}+\cdots\right)\right| \\
& =\left|\frac{2 f^{\prime}\left(u_{j}^{n}\right)}{I^{2}\left(u^{n}\right)} u_{x_{j}}^{n}-\frac{2 f^{\prime}\left(u_{j}^{n}\right)}{I_{h}^{2}\left(u^{n}\right)} u_{x_{j}}^{n}\right|+O\left(\Delta x^{2}\right) \\
& \leqslant c_{2}\left|\frac{1}{I^{2}\left(u^{n}\right)}-\frac{1}{I_{h}^{2}\left(u^{n}\right)}\right|+O\left(\Delta x^{2}\right) \leqslant O\left(\Delta x^{2}\right),
\end{aligned}
$$


for some constant $c_{2}=2 M_{1} M_{2}$, with $M_{2}=\sup u_{x}, x \in[0,1]$. Finally

$$
\begin{aligned}
\left|G_{3}\left(u_{j}^{n}\right)-G_{3 h}\left(u_{j}^{n}\right)\right|= & \mid 2 \frac{f\left(u_{j}^{n}\right)}{I^{3}\left(U_{j}^{n}\right)}\left(\lambda \frac{I_{1}\left(u^{n}\right)}{\left.I^{2}\left(u^{n}\right)\right)}-\left(f\left(u_{J}^{n}\right)-f\left(u_{0}^{n}\right)\right)\right. \\
& -2 \frac{f\left(u_{j}^{n}\right)}{I_{h}^{3}\left(u_{j}^{n}\right)}\left(\lambda \frac{I_{1 h}\left(u^{n}\right)}{\left.I_{h}^{2}\left(u^{n}\right)\right)}-\left(f\left(u_{J}^{n}\right)-f\left(u_{0}^{n}\right)\right) \mid,\right. \\
\leqslant & c_{3}\left(\left|\frac{1}{I_{h}^{2}\left(u^{n}\right)}-\frac{1}{I^{2}\left(u^{n}\right)}\right|+\left|I_{1}\left(u^{n}\right)-I_{1 h}\left(u^{n}\right)\right|+\left|I\left(u^{n}\right)-I_{h}\left(u^{n}\right)\right|\right) \\
\leqslant & O\left(\Delta x^{2}\right)
\end{aligned}
$$

for $c_{3}=\max \left\{\frac{2 \lambda f(0)\left(1+M_{1} f(0) f^{2}\left(M_{u}\right)\right)}{f^{5}\left(M_{u}\right)}, \frac{6 \lambda f^{4}(0) M_{1}\left(1+f(0) f^{2}\left(M_{u}\right)\right)}{f^{8}\left(M_{u}\right)}\right\}$. The last inequality comes by adding and subtracting the term $2 \frac{f\left(u_{j}^{n}\right)}{I^{3}\left(u_{j}^{n}\right)}\left(\lambda \frac{I_{1 h}\left(u^{n}\right)}{\left.I_{h}^{2}\left(u^{n}\right)\right)}-\left(f\left(u_{J}^{n}\right)-f\left(u_{0}^{n}\right)\right)\right.$, the fact that $\mid \frac{f\left(u_{j}^{n}\right)}{I^{3}\left(U_{j}^{n}\right)}-$ $\frac{f\left(u_{j}^{n}\right)}{I_{h}^{3}\left(U_{j}^{n}\right)} \mid \leqslant O\left(\Delta x^{2}\right)$ and that $\left|\frac{I_{1}\left(u^{n}\right)}{\left.I^{2}\left(u^{n}\right)\right)}-\frac{I_{1 h}\left(u^{n}\right)}{\left.I_{h}^{2}\left(u^{n}\right)\right)}\right| \leqslant O\left(\Delta x^{2}\right)$. Therefore $\left|G\left(u_{j}^{n}\right)-G_{h}\left(u_{j}^{n}\right)\right| \leqslant$ $O\left(\Delta x^{2}\right)$. Thus we obtain that for $1 \leqslant n \leqslant N,\left|\tau_{j}^{n}\right|=O\left(\Delta t^{2}+\Delta x^{2}\right)$. In addition

$$
\begin{aligned}
\left|\tau_{J}^{n+1}\right|= & \frac{1}{\Delta t} \mid\left(1-\frac{3 r}{2}+\frac{r^{2}}{2}\right) u_{J}^{n}+\left(2 r-r^{2}\right) u_{J-1}^{n}-\frac{r}{2}(1-r) u_{J-2}^{n}+\lambda \Delta t F_{h}\left(u_{J}^{n}\right) \\
= & \frac{1}{\Delta t} \mid\left(1-\frac{3 r}{2}+\frac{r^{2}}{2}\right) u_{J}^{n} \\
& +\left(2 r-r^{2}\right)\left(u_{J}^{n}\right)-u_{J}^{n+1} \mid \\
& -\frac{r}{2}(1-r)\left(u_{J}^{n}-2 \Delta u_{x J}^{n}+\frac{\Delta x^{2}}{2} u_{x x J}^{n}-\frac{\Delta x^{3}}{6} u_{x x x_{j}}^{n}+\cdots\right) \\
& \left.+\lambda u_{x J}^{n}+2 \Delta x^{2} u_{x x J}^{n}-\frac{4 \Delta x^{3}}{3} u_{x x x J}^{n}+\cdots\right) \\
& -\left(u_{J}^{n}+\Delta t F_{t J}^{n}+\frac{\Delta t^{2}}{2} u_{t t J}^{n}+\frac{\Delta t^{3}}{6} u_{t t t J}^{n}+\cdots\right) \mid .
\end{aligned}
$$

or in a similar way as before

$$
\begin{aligned}
\left|\tau_{J}^{n+1}\right|= & \frac{1}{\Delta t} \mid-\Delta t\left(u_{t J}^{n}+u_{x J}^{n}\right)+\lambda \Delta t F_{h}\left(u_{J}^{n}\right)+\lambda \frac{\Delta t^{2}}{2} G\left(u_{J}^{n}\right)-\frac{\Delta t^{2}}{2} u_{t t_{J}}^{n} \\
& +\frac{\Delta t^{2}}{2} u_{x x J}^{n}+\frac{\Delta t \Delta x^{2}}{3} u_{x x x J}^{n}+\frac{\Delta t^{3}}{6} u_{t t t J}^{n}+\cdots \mid \\
\leqslant & \lambda\left|F_{h}\left(u_{J}^{n}\right)-F\left(u_{J}^{n}\right)\right|+\lambda\left|G\left(u_{J}^{n}\right)-G_{h}\left(u_{J}^{n}\right)\right| \\
= & +\frac{\Delta x^{2}}{3}\left|u_{x x x J}^{n}\right|+\frac{\Delta t^{2}}{6}\left|u_{t t t J}^{n}\right|+\cdots \\
= &
\end{aligned}
$$

Therefore we have that $\left|\tau_{j}^{n}\right|=O\left(\Delta t^{2}+\Delta x^{2}\right)$, for $j=0, \ldots, J, \quad n=1, \ldots, N$ and hence, using also the assumption on the initial condition, that the scheme is consistent and $\lim _{\Delta x, \Delta t \rightarrow 0}\left\|\phi_{h}\left(u_{h}\right)\right\|_{Y}=\lim _{\Delta x, \Delta t \rightarrow 0}\left\|l_{h}\right\|_{Y}=0$. 


\section{Stability}

Proposition 5. Under the hypotheses of proposition (4) the discretization (2.1) is stable for $r=\frac{\Delta t}{\Delta x} \leqslant 1$.

Proof. Let $V, W \in B\left(u_{h}, M_{h}\right)$ of $X$ with $\phi_{h}(V)=Z$ and $\phi_{h}(W)=S$. We set $E^{n}=$ $V^{n}-W^{n} \in \mathbb{R}^{J+1}, 0 \leqslant n \leqslant N$. We have for $j=1, \ldots, J-1$ that

$$
\begin{aligned}
\left|E_{j}^{n+1}\right|=\quad & \mid \frac{r}{2}(1+r) V_{j-1}^{n}+\left(1-r^{2}\right) V_{j}^{n}-\frac{r}{2}(1-r) V_{j+1}^{n}+\lambda \Delta t F_{h}\left(V_{j}^{n}\right) \\
& +\lambda \frac{\Delta t^{2}}{2} G\left(V_{j}^{n}\right) \\
& -\frac{r}{2}(1+r) W_{j-1}^{n}-\left(1-r^{2}\right) W_{j}^{n}+\frac{r}{2}(1-r) W_{j+1}^{n}-\lambda \Delta t F_{h}\left(W_{j}^{n}\right) \\
& -\lambda \frac{\Delta t^{2}}{2} G\left(W_{j}^{n}\right)-\Delta t\left(Z_{j}^{n+1}-S_{j}^{n+1}\right) \mid \\
\leqslant \quad & \frac{r}{2}(1+r)\left|E_{j-1}^{n}\right|+\left(1-r^{2}\right)\left|E_{j}^{n}\right|+\frac{r}{2}(1-r)\left|E_{j+1}^{n}\right| \\
& +\lambda \Delta t\left|F_{h}\left(V_{j}^{n}\right)-F_{h}\left(W_{j}^{n}\right)\right|+\lambda \frac{\Delta t^{2}}{2}\left|G_{h}\left(V_{j}^{n}\right)-G_{h}\left(W_{j}^{n}\right)\right| \\
& +\Delta t\left|\left(Z_{j}^{n+1}-S_{j}^{n+1}\right)\right| .
\end{aligned}
$$

We have, as it is shown in proposition (2), that $\left|F_{h}\left(V_{j}^{n}\right)-F_{h}\left(W_{j}^{n}\right)\right| \leqslant c_{1}\left|E_{j}^{n}\right|+c_{2}|| E^{n} \|_{1}$, $j=1, \ldots, J$. Then regarding the term $\left|G_{h}\left(V_{j}^{n}\right)-G_{h}\left(W_{j}^{n}\right)\right|$ we obtain

$$
\begin{aligned}
& \left|G_{1 h}\left(V_{j}^{n}\right)-G_{1 h}\left(W_{j}^{n}\right)\right|=\lambda\left|\frac{f^{\prime}\left(V_{j}^{n}\right) f\left(V_{j}^{n}\right)}{I_{h}^{4}\left(V^{n}\right)}-\frac{f^{\prime}\left(W_{j}^{n}\right) f\left(W_{j}^{n}\right)}{I_{h}^{4}\left(W^{n}\right)}\right| \\
\leqslant & \lambda\left|\frac{f^{\prime}\left(V_{j}^{n}\right)}{I_{h}^{2}\left(V^{n}\right)}\left(F_{h}\left(V_{j}^{n}\right)-F_{h}\left(W_{j}^{n}\right)\right)\right| \\
& +\lambda\left|\frac{f^{\prime}\left(W_{j}^{n}\right)}{I_{h}^{2}\left(W_{j}^{n}\right)}\left[\frac{1}{I_{h}\left(V^{n}\right)}\left(f^{\prime}\left(V_{j}^{n}\right)-f^{\prime}\left(W_{j}^{n}\right)\right)+f^{\prime}\left(W_{j}^{n}\right)\left(\frac{1}{I_{h}^{2}\left(V_{j}^{n}\right)}-\frac{1}{I_{h}^{2}\left(W_{j}^{n}\right)}\right)\right]\right| \\
\leqslant & c_{3}\left|E_{j}^{n}\right|+c_{4}|| E^{n} \|_{1},
\end{aligned}
$$

where here $c_{3}=\max \left\{\frac{\lambda M_{1} L}{f^{4}\left(M_{h}\right)}, \frac{\lambda f(0) L^{\prime}}{f^{4}\left(M_{h}\right)}\right\}$ and $c_{4}=\frac{2 \lambda M_{1} f^{2}(0) L}{f^{6}\left(M_{h}\right)}$. Also

$$
\begin{aligned}
& \left|G_{2 h}\left(V_{j}^{n}\right) \frac{V_{j+1}^{n}-V_{j-1}^{n}}{2 \Delta x}-G_{2 h}\left(W_{j}^{n}\right) \frac{W_{j+1}^{n}-W_{j-1}^{n}}{2 \Delta x}\right| \\
& =\frac{1}{2 \Delta x}\left|G_{2 h}\left(V_{j}^{n}\right)\left(E_{j+1}^{n}-E_{j-1}^{n}\right)+\left(W_{j+1}^{n}-W_{j-1}^{n}\right)\left(G_{2}\left(V_{j}^{n}\right)-V_{2}\left(W_{j}^{n}\right)\right)\right| \\
& \leqslant \frac{1}{2 \Delta x}\left[c_{5}\left(\left|E_{j+1}^{n}\right|+\left|E_{j}^{n}\right|+\left|E_{j-1}^{n}\right|\right)+c_{6}|| E^{n}||_{1}\right],
\end{aligned}
$$

where here $c_{5}=\max \frac{2 M_{1}}{f^{2}\left(M_{h}\right)}, \frac{4 L^{\prime} M_{h}}{f^{2}\left(M_{h}\right)}, c_{6}=\frac{8 M_{h} M_{1} f(0) L}{f^{4}\left(M_{h}\right)}$. Finally

$$
\begin{aligned}
\left|G_{3 h}\left(V_{j}^{n}\right)-G_{3 h}\left(W_{j}^{n}\right)\right|= & \mid-2 \frac{f\left(V_{j}^{n}\right)}{I_{h}^{3}\left(V^{n}\right)}\left(\lambda \frac{I_{1 h}\left(V^{n}\right)}{I_{h}^{2}\left(V^{n}\right)}-f\left(V_{J}^{n}\right)+f\left(V_{0}^{n}\right)\right) \\
& +2 \frac{f\left(W_{j}^{n}\right)}{I_{h}^{3}\left(W^{n}\right)}\left(\lambda \frac{I_{1 h}\left(W^{n}\right)}{I_{h}^{2}\left(W^{n}\right)}-f\left(W_{J}^{n}\right)+f\left(W_{0}^{n}\right)\right) \\
\leqslant & \left.c_{7}\left|E_{j}^{n}\right|+c_{8}|| E^{n} \|_{1}\right) \leqslant c_{7}\left|E_{j}^{n}\right|+c_{8}|| E^{n} \|_{1} .
\end{aligned}
$$


In this case $c_{8}=\max \left\{\frac{4 \lambda f^{3}(0) M_{1} L}{f^{7}\left(M_{h}\right)}, \frac{2 \lambda f(0) L}{f^{3}\left(M_{h}\right)}, \frac{2 \lambda f(0) L\left(M_{1} L+f(0) L^{\prime}\right)}{f^{5}\left(M_{h}\right)}, \frac{6 \lambda f^{4}(0) M_{1} L}{f^{8}\left(M_{h}\right)}, \frac{6 f^{4}(0) L}{f^{6}\left(M_{h}\right)},\right\}$ and $c_{7}=$ $\frac{2 L f(0)}{f^{3}\left(M_{h}\right)}\left(\frac{\lambda M_{1}}{f^{2}\left(M_{h}\right)}+1\right)$. Note also that here was used the fact that $\left.\mid I_{1 h}\left(V^{n}\right)\right)-I_{1 h}\left(W^{n}\right) \mid \leqslant$ $\left(M_{1} L+f(0) L^{\prime}\right)|| E^{n} \|_{1}$ and that $\left|E_{J}^{n}\right| \leqslant\left\|E^{n}\right\|_{1}$.

Thus, by denoting $C_{1}^{\prime}=\max \left\{c_{1}, c_{2}, \frac{\lambda}{4} r c_{5}, \frac{\lambda}{4} r c_{6}\right\}, C_{2}=\frac{\lambda}{2} \max \left\{c_{3}, c_{4}, c_{7}, c_{8}\right\}$ and given that $r \leqslant 1$ we obtain

$$
\begin{aligned}
\left|E_{j}^{n+1}\right| \leqslant & {\left[\frac{r}{2}(1+r)+C_{1}^{\prime} \Delta t\right]\left|E_{j-1}^{n}\right|+\left[\left(1-r^{2}\right)+C_{1}^{\prime} \Delta t+C_{2} \Delta t^{2}\right]\left|E_{j}^{n}\right|+} \\
& +\left[\frac{r}{2}(1-r)+C_{1}^{\prime} \Delta t\right]\left|E_{j+1}^{n}\right|+\left[C_{1}^{\prime} \Delta t+C_{2} \Delta t^{2}\right]|| E^{n} \|_{1}+\Delta t\left|\left(Z_{j}^{n+1}-S_{j}^{n+1}\right)\right| .
\end{aligned}
$$

for $1 \leqslant j \leqslant J-1$. In a similar way we deduce that

$$
\begin{aligned}
\left|E_{J}^{n+1}\right| \leqslant & \left(1-\frac{3 r}{2}+\frac{r^{2}}{2}\right)\left|E_{J}^{n}\right|+\left(2 r-r^{2}\right)\left|E_{J-1}^{n}\right|+\frac{r}{2}(1-r)\left|E_{J-2}^{n}\right|+\lambda \Delta t\left|F_{h}\left(V_{J}^{n}\right)-F_{h}\left(W_{J}^{n}\right)\right| \\
& +\lambda \frac{\Delta t^{2}}{2}\left|G_{h}\left(V_{J}^{n}\right)-G_{h}\left(W_{J}^{n}\right)\right|+\Delta t\left|\left(Z_{J}^{n+1}-S_{J}^{n+1}\right)\right|,
\end{aligned}
$$

or

$$
\begin{aligned}
\left|E_{J}^{n+1}\right| \leqslant & {\left[\left(1-\frac{3 r}{2}+\frac{r^{2}}{2}\right)+C_{1}^{\prime \prime} \Delta t+C_{2} \Delta t^{2}\right]\left|E_{J}^{n}\right|+\left[\left(2 r-r^{2}\right)+C_{1}^{\prime \prime} \Delta t\right]\left|E_{J-1}^{n}\right| } \\
& +\left[\frac{r}{2}(1-r)+C_{1}^{\prime \prime} \Delta t\right]\left|E_{J-2}^{n}\right| \\
& +\left.\left[C_{1}^{\prime \prime} \Delta t+C_{2} \Delta t^{2}\right]|| E^{n}\right|_{1}+\Delta t\left|\left(Z_{J}^{n+1}-S_{J}^{n+1}\right)\right|,
\end{aligned}
$$

where $C_{1}^{\prime \prime}=\max \left\{c_{1}, c_{2}, c_{9}, c_{10}\right\}, c_{9}=\max \left\{\frac{8 M_{1}}{f^{2}\left(M_{h}\right)}, \frac{64 M_{1} L^{\prime}}{f^{2}\left(M_{h}\right)}\right\}, c_{10}=\frac{32 f(0) M_{h} M_{1} L}{f^{4}\left(M_{h}\right)}$.

Note that the terms $\left|E_{j}^{n}\right|, j=1, \ldots, J-1$ can be bounded in the following way

$$
\begin{aligned}
\left|E_{j}^{n+1}\right| \leqslant & 2 r\left|E_{j-1}^{n}\right|+\left[\left(1-r^{2}\right)+C_{1}^{\prime} \Delta t+C_{2} \Delta t^{2}\right]\left|E_{j}^{n}\right|+\left[\frac{r}{2}(1-r)+C_{1}^{\prime} \Delta t\right]\left|E_{j+1}^{n}\right| \\
& +\left[C_{1}^{\prime} \Delta t+C_{2} \Delta t^{2}\right]|| E^{n} \|_{1}+\Delta t\left|\left(Z_{j}^{n+1}-S_{j}^{n+1}\right)\right| .
\end{aligned}
$$

Then observing that :

$\frac{r}{2}(1+r)\left|E_{J-2}^{n}\right|+\left(2 r-r^{2}\right)\left|E_{J-1}^{n}\right|+\frac{r}{2}\left(1-r^{2}\right)\left|E_{J-2}^{n}\right|=r\left|E_{J-2}^{n}\right|+\left(2 r-r^{2}\right)\left|E_{J-1}^{n}\right|$ $\leqslant 2 r\left(\left|E_{J-2}^{n}\right|+\left|E_{J-1}^{n}\right|+\left|E_{J}^{n}\right|\right)$ and that $\left(1-r^{2}\right)\left|E_{J-1}^{n}\right|+\left(1-\frac{3 r}{2}+\frac{r^{2}}{2}\right)\left|E_{J}^{n}\right| \leqslant\left(1-r^{2}\right)\left(\left|E_{J-1}^{n}\right|+\left|E_{J}^{n}\right|\right)$, we can combine equations (3.2) and (3.3), with $C_{1}=\max \left\{C_{1}^{\prime}, C_{1}^{\prime \prime}\right\}$, in order to obtain

$$
\begin{aligned}
\left\|E^{n+1}\right\|_{1} \leqslant & {\left[2 r+C_{1} \Delta t\right]\left\|E^{n}\right\|_{1}+\left[\left(1-r^{2}\right)+C_{1} \Delta t+C_{2} \Delta t^{2}\right]\left\|E^{n}\right\|_{1} } \\
+ & {\left[\frac{r}{2}(1-r)+C_{1} \Delta t\right]\left\|E^{n}\right\|_{1}+\Delta t\left\|\left(Z^{n+1}-S^{n+1}\right)\right\|_{1} . }
\end{aligned}
$$

or

$$
\left\|E^{n+1}\right\|_{1} \leqslant\left[\left(1+\frac{5 r}{2}-\frac{3 r^{2}}{2}\right)+C_{1} \Delta t+C_{2} \Delta t^{2}\right]\left\|E^{n}\right\|_{1}+\Delta t\left\|\left(Z^{n+1}-S^{n+1}\right)\right\|_{1} .
$$

Therefore by a standard recursive argument we obtain

$$
\max _{0 \leqslant n \leqslant N}\left\|E^{n}\right\|_{1} \leqslant C\left(\left\|E^{0}\right\|_{1}+\Delta t \sum_{n=1}^{N}\left\|\left(Z^{n}-S^{n}\right)\right\|_{1}\right)=C\left\|\phi_{h}(V)-\phi_{h}(W)\right\|_{Y},
$$

for some constant $C$. Thus $\|V-W\|_{X} \leqslant C\left\|\phi_{h}(V)-\phi_{h}(W)\right\|_{Y}$, and the scheme is stable. 


\section{Convergence}

Regarding the convergence of the scheme we have the following proposition:

Proposition 6. Assuming that the hypotheses of proposition (4) hold and that $U^{0}$ is such that $\left\|U^{0}-u^{0}\right\|_{1}=O\left(\Delta x^{2}\right)$ as $\Delta x \rightarrow 0$, then the numerical solution of the scheme $U_{h}$ satisfies

$$
\left\|U_{h}-u_{h}\right\|_{X}=O\left(\Delta t^{2}+\Delta x^{2}\right)
$$

and

$$
\left\|U_{h}-u_{h}\right\|_{\infty}=O\left(\Delta t^{2}+\Delta x^{2}\right)
$$

as $\Delta x, \Delta t \rightarrow 0$.

Proof. Given that $\left\|U^{0}-u^{0}\right\|_{1}=O\left(\Delta x^{2}\right)$ and using the same arguments as in proposition (3) we have that $\left\|U_{h}-u_{h}\right\|_{X}=O\left(\Delta t^{2}+\Delta x^{2}\right)$.

The relation

$$
\left\|U_{h}-u_{h}\right\|_{\infty}=\max _{0 \leqslant n \leqslant N}\left\|e^{n}\right\|_{\infty}=O\left(\Delta t^{2}+\Delta x^{2}\right),
$$

also holds. Indeed for $e^{n}=U_{h}{ }^{n}-u_{h}{ }^{n}$ we have $\left|e_{0}^{n}\right|=0$, for $1 \leqslant n \leqslant N$ and

$$
e_{j}^{n+1}=U_{j}^{n+1}-u_{j}^{n+1}=L\left(U_{j}^{n}\right)-L\left(u_{j}^{n}\right)+L\left(u_{j}^{n}\right)-u_{j}^{n+1},
$$

for $0 \leqslant n \leqslant N-1$, where

$$
\begin{aligned}
& L\left(U_{j}^{n}\right):=\frac{r}{2}(1+r) U_{j-1}^{n}+\left(1-r^{2}\right) U_{j}^{n}-\frac{r}{2}(1-r) U_{j+1}^{n} \\
&+\lambda \Delta t F_{h}\left(U_{j}^{n}\right)+\lambda \frac{\Delta t^{2}}{2} G_{h}\left(U_{j}^{n}\right), \quad j=1, \ldots, J-1, \\
& L\left(U_{J}^{n}\right):=\left(1-\frac{3 r}{2}+\frac{r^{2}}{2}\right) U_{J}^{n}+\left(2 r-r^{2}\right) U_{J-1}^{n}-\frac{r}{2}(1-r) U_{J-2}^{n} \\
&+\lambda \Delta t F_{h}\left(U_{J}^{n}\right)+\lambda \frac{\Delta t^{2}}{2} G_{h}\left(U_{J}^{n}\right) .
\end{aligned}
$$

Hence

$$
\left|e_{j}^{n+1}\right| \leqslant\left|L\left(U_{j}^{n}\right)-L\left(u_{j}^{n}\right)\right|+\Delta t\left|\tau_{j}^{n+1}\right|
$$

for $j=1, \ldots, J$. Then following a similar procedure as for the derivation of equations $(3.2-3.3)$ we obtain

$$
\begin{aligned}
&\left|L\left(U_{j}^{n}\right)-L\left(u_{j}^{n}\right)\right| \leqslant \frac{r}{2}(1+r)\left|e_{j-1}^{n}\right|+\left[\left(1-r^{2}\right)\right]\left|e_{j}^{n}\right|-\frac{r}{2}(1-r)\left|e_{j+1}^{n}\right| \\
&+\lambda \Delta t\left|F_{h}\left(U_{j}^{n}\right)-F_{h}\left(u_{j}^{n}\right)\right|+\lambda \frac{\Delta t^{2}}{2}\left|G_{h}\left(U_{j}^{n}\right)-G_{h}\left(u_{j}^{n}\right)\right|, j=1, \ldots, J-1, \\
&\left|L\left(U_{J}^{n}\right)-L\left(u_{J}^{n}\right)\right| \leqslant\left(1-\frac{3 r}{2}+\frac{r^{2}}{2}\right)\left|e_{J}^{n}\right|+\left(2 r-r^{2}\right)\left|e_{J-1}^{n}\right|-\frac{r}{2}(1-r)\left|e_{J-2}^{n}\right| \\
&+\lambda \Delta t\left|F_{h}\left(U_{J}^{n}\right)-F_{h}\left(u_{J}^{n}\right)\right|+\lambda \frac{\Delta t^{2}}{2}\left|G_{h}\left(U_{J}^{n}\right)-G_{h}\left(u_{J}^{n}\right)\right| .
\end{aligned}
$$

Thus for a constant $C_{0}>\left(1+\frac{5 r}{2}-\frac{3 r^{2}}{2}\right)$ we have

$$
\left|L\left(U_{j}^{n}\right)-L\left(u_{j}^{n}\right)\right| \leqslant\left(C_{0}+C_{1} \Delta t+C_{2} \Delta t^{2}\right)\left\|e^{n}\right\|_{1}, \quad j=1, \ldots, J .
$$


Hence by equation (3.4) we obtain

$$
\left\|e^{n}\right\|_{\infty} \leqslant\left(C_{0}+C_{1} \Delta t+C_{2} \Delta t^{2}\right)\left\|e^{n}\right\|_{1}+O\left(\Delta t^{2}+\Delta x^{2}\right) .
$$

On the other hand we can also derive the relation

$$
\left\|e^{n+1}\right\|_{1} \leqslant\left(C_{0}+C_{1} \Delta t+C_{2} \Delta t^{2}\right)\left\|e^{n}\right\|_{1}+O\left(\Delta t^{2}+\Delta x^{2}\right) .
$$

By the relations (3.5) and (3.6) we deduce that, for some constant $C$,

$$
\left\|e^{n}\right\|_{\infty} \leqslant C\left\|e^{0}\right\|_{1}+O\left(\Delta t^{2}+\Delta x^{2}\right)
$$

for every $n, 1 \leqslant n \leqslant N$ and

$$
\max _{O \leqslant n \leqslant N}\left\|e^{n}\right\|_{\infty} \leqslant C\left\|e^{0}\right\|_{1}+O\left(\Delta t^{2}+\Delta x^{2}\right) .
$$

Finally, provided that $\left\|e^{0}\right\|_{1}=O\left(\Delta x^{2}\right)$, we have

$$
\max _{O \leqslant n \leqslant N}\left\|e^{n}\right\|_{\infty}=O\left(\Delta t^{2}+\Delta x^{2}\right) .
$$

Remark Note that in the above Lax-Wendroff scheme omitting the term $\lambda \frac{\Delta t^{2}}{2} G(u)$ will result in a scheme that is of order $O\left(\Delta t+\Delta x^{2}\right)$. This can be easily seen if in the relevant proofs is set $G(u)=0$. Moreover, a modification of the scheme (3.1) can be obtained by using the Beam Warming approximation for $j=2, \ldots, J$ and the Lax-Wendroff approximation for $j=1$. Such a scheme will have the same stability properties as (3.1).

\section{A high Resolution Scheme}

In the following, motivated by the analysis in [18], we will introduce a high resolution method. This method will allow for better behaviour of the numerical solution near discontinuities by the use of the upwind method and higher order of accuracy in smooth parts of the solution by the use of the Lax-Wendroff or the Beam-Warming method. For simplicity we will consider the Lax-Wendroff approach only for the linear part of the equation (i.e. setting $G=0$ in (3.1) ) which gives accuracy $O\left(\Delta t+\Delta x^{2}\right)$ for the smooth parts of the solution.

We can construct a finite volume scheme by integrating equation (1.1a) over the set $\left[x_{j-\frac{1}{2}}, x_{j+\frac{1}{2}}\right] \times\left[t_{n}, t_{n+1}\right]$, where $x_{j+a}:=x_{j}+a \Delta x$. Indeed in such a way we obtain

$$
\begin{aligned}
\int_{x_{j-\frac{1}{2}}}^{x_{j+\frac{1}{2}}}\left(u\left(x, t_{n+1}\right)-u\left(x, t_{n}\right)\right) d x & =-\int_{t_{n}}^{t_{n+1}}\left(u\left(x_{j+\frac{1}{2}}, t\right)-u\left(x_{j-\frac{1}{2}}, t\right)\right) d t \\
& +\lambda \int_{t_{n}}^{t_{n+1}} \int_{x_{j-\frac{1}{2}}}^{x_{j+\frac{1}{2}}} F(u(x, t)) d x d t .
\end{aligned}
$$

Equation (3.7) defines a weak solution for problem (1.1). As a next step we consider numerical methods of the form

$$
U_{j}^{n+1}=U_{j}^{n}-r\left[\Phi\left(U^{n} ; j\right)-\Phi\left(U^{n} ; j-1\right)\right]+\lambda \Delta t F_{h}\left(U_{j}^{n}\right),
$$

where $\Phi\left(u^{n} ; j\right):=\Phi\left(U_{j-2}^{n}, U_{j-2}^{n}, U_{j-1}^{n}, U_{j}^{n}, U_{j+1}^{n}\right) . \Phi\left(U^{n} ; j\right)=U_{j}^{n}$ for the upwind method, $\Phi\left(U^{n} ; j\right)=\frac{1}{2}\left(U_{j+1}^{n}+U_{j}^{n}\right)-\frac{r}{2}\left(U_{j+1}^{n}-U_{j}^{n}\right)$ for the Lax-Wendroff method and for the Beam 
Warming method $\Phi\left(U^{n} ; j\right)=U_{j}^{n}+\frac{1}{2}(1-r)\left(U_{j}^{n}-U_{j-1}^{n}\right)$. Then method (3.8) is consistent with equation (3.7) if $\Phi$ reduces to the true homogeneous flux for constant flow.

In order to investigate the stability of this method we need the following definitions. For a grid function $U^{n} \in \mathbb{R}^{J+1}$ we define the total variation by $T V\left(U^{n}\right):=\sum_{j=1}^{J}\left|U_{j}^{n}-U_{j-1}^{n}\right|$. We can also extend the grid function $U_{h} \in X$ by defining a piecewise constant function $u_{h}(x, t):=U_{j}^{n}$ for $(x, t) \in\left[x_{j-\frac{1}{2}}, x_{j+\frac{1}{2}}\right) \times\left[t_{n}, t_{n+1}\right)$, for $r$ fixed. In this case, for $u_{h}\left(x, t_{n}\right)=u_{h}^{n}$, $T V\left(u_{h}^{n}\right)=T V\left(U^{n}\right)$. Also we define the total variation $T V_{T}\left(U_{h}\right)$, of $U_{h} \in X$ in both space and time, in the following way : $T V_{T}\left(U_{h}\right):=\sum_{n=0}^{[T / \Delta t]} \sum_{j=1}^{J}\left[\Delta t\left|U_{j}^{n}-U_{j-1}^{n}\right|+\Delta x\left|U_{j}^{n+1}-U_{j}^{n}\right|\right]=$ $\sum_{n=0}^{[T / \Delta t]}\left(\Delta t T V\left(U^{n}\right)+\left\|U^{n+1}-U^{n}\right\|_{1}\right)$, and $T V_{T}\left(u_{h}\right)=T V_{T}(U)$. Note that the set $\mathcal{K}_{T}:=$ $\left\{v \in L_{1, T}([0,1]): T V_{T}(v) \leqslant R, R>0\right\}$ is a compact subset of $L_{1 T}([0,1]):=\{v,[0,1] \rightarrow \mathbb{R},:$ $\left.\|v\|_{1 T}:=\int_{0}^{T} \int_{0}^{1} v(x, t) d x d t<\infty\right\}$.

Proposition 7. For a method of the general form (3.8) if the numerical homogeneous flux $\Phi$ is Lipschitz continuous, $r<1$ and for the initial data $U^{0}$ we have $T V\left(U^{0}\right)<\infty$, then the method is TV-stable.

Proof. We know (Theorem 12.2 in [16]) that for a numerical method with Lipschitz continuous numerical flux, if for any initial data $u^{0}$ there exist some $\Delta t_{0}$ and $R>0$ such that $T V\left(U^{n}\right)<R$ for every $n$ and $\Delta t$ with $\Delta t<\Delta t_{0}, n \Delta t \leqslant T$, then the method is $T V$-stable.

In our case initially we have to show that the relation $T V\left(U^{n}\right) \leqslant R$ (and consequently $\left|U^{n}\right| \leqslant \frac{R}{2}$ ) implies that $\left\|U^{n+1}-U^{n}\right\|_{1} \leqslant c \Delta t$. By equation (3.8) we have

$$
\begin{aligned}
\left\|U^{n+1}-U^{n}\right\|_{1} & =\Delta t\left[\sum_{j=1}^{J}\left|\Phi_{j+\frac{1}{2}}^{n}-\Phi_{j-\frac{1}{2}}^{n}\right|+\lambda \Delta x F_{h}\left(U_{j}^{n}\right)\right] \\
& \leqslant \Delta t\left[K \sum_{j=1}^{J} \sum_{i=-2}^{1}\left|\Phi_{j+\frac{1}{2}}^{n}-\Phi_{j-\frac{1}{2}}^{n}\right|+\lambda \sum_{j=1}^{J} \Delta x F_{h}\left(U_{j}^{n}\right)\right] \leqslant c \Delta t,
\end{aligned}
$$

for $c=4 K R+\frac{\lambda f(0)}{f^{2}(R / 2)}$.

Thus now it is sufficient to show that $T V\left(U^{n}\right) \leqslant R$ which can be implied by the relation $T V\left(U^{n+1}\right)<\left(c_{1}+c_{2} \Delta t\right) T V\left(U^{n}\right)$ for some constants $c_{1}, c_{2}$ independent of $\Delta t$. Note also that according to [15] discontinuities in the initial condition are simply propagate along the characteristics and even in this case the solution remains bounded if $u^{0}$ is bounded. Thus we may assume, by consistency, that $U^{n}$ is also bounded by some constant M. We have

$$
\begin{aligned}
\sum_{j=1}^{J}\left|U_{j}^{n+1}-U_{j-1}^{n+1}\right| & =\sum_{j=1}^{J} \mid U_{j}^{n}-r\left[\Phi\left(U^{n} ; j\right)-\Phi\left(U^{n} ; j-1\right)\right]+\lambda \Delta t F_{h}\left(U_{j}^{n}\right) \\
& \quad-U_{j-1}^{n}+r\left[\Phi\left(U^{n} ; j-1\right)-\Phi\left(U^{n} ; j-2\right)\right]-\lambda \Delta t F_{h}\left(U_{j-1}^{n}\right) \mid \\
& \leqslant \sum_{j=1}^{J}\left[\left|U_{j}^{n}-U_{j-1}^{n}\right|+r c_{1} \sum_{i=-2}^{1}\left|U_{j+i}^{n}-U_{j+i-1}^{n}\right|+c_{2}\left|U_{j}^{n}-U_{j-1}^{n}\right|\right] \\
\leqslant & \left(c_{3}+c_{2} \Delta t\right) \sum_{j=1}^{J}\left|U_{j}^{n}-U_{j-1}^{n}\right|,
\end{aligned}
$$

for some constant $c_{1}, c_{2}=\frac{\lambda L}{f^{2}(M)}$, and $c_{3}=1+4 r c_{2}$. Hence method (3.8) is $T V$-stable. 
Therefore a method of the form (3.8), which generates a numerical solution $U_{h}$, consistent with the conservation law (3.7) is convergent to an element $w \in \mathcal{K}_{T}$. By the Lax-Wendroff theorem we know that $w$ is also a weak solution of (3.7) ([16]). The method converges in the sense that $\operatorname{dist}(U, \mathcal{W}) \rightarrow 0$ as $\Delta t \rightarrow 0$ for $\mathcal{W}=\{w, w$ is a weak solution of (3.7) $\}$, $\operatorname{dist}(U, \mathcal{W}):=\inf _{w \in \mathcal{W}}\|U-w\|$ (Theorem 12.3 in [16]).

In the following we can introduce a high resolution method by specifying the form of $\Phi$ with the use of appropriate limiters. We consider a specific form of (3.8) :

$$
\begin{aligned}
U_{j}^{n+1}= & U_{j}^{n}-r\left(U_{j}^{n}-U_{j-1}^{n}\right)+\lambda \Delta t f_{h}\left(U_{j}^{n}\right) \\
& -\frac{1}{2} r(1-r)\left[\phi\left(\theta_{j+\frac{1}{2}}\right)\left(U_{j+1}^{n}-U_{j}^{n}\right)+\phi\left(\theta_{j-\frac{1}{2}}\right)\left(U_{j}^{n}-U_{j-1}^{n}\right)\right] .
\end{aligned}
$$

The limiter $\phi\left(\theta_{j}\right)$ is defined for the minmod method in the following way

$$
\phi(\theta)=\operatorname{minmod}(1, \theta)=\left\{\begin{array}{ccc}
1, & \text { for } \quad 1<|\theta|, & \theta>0 \\
\theta, & \text { for } \quad|\theta|<1, & \theta>0 \\
0 & \text { for } & \theta \leqslant 0
\end{array}\right.
$$

for $j=2, \ldots, J-2$ and $\theta_{j}^{n}:=\frac{U_{j-1}^{n}-U_{j-2}^{n}}{U_{j}^{n}-U_{j-1}^{n}}$. Note that for $\phi(\theta)=1$ we take the Lax-Wendroff method, for $\phi(\theta)=\theta$ the Beam Warming method and for $\phi(\theta)=0$ the upwind method. To ensure that for $j=2$ we have the Lax-Wendroff approximation and that for $j=J$ the Beam Warming approximation we set $\phi\left(\theta_{1+\frac{1}{2}}\right)=\phi\left(\theta_{\frac{1}{2}}\right)=\max \{0, \operatorname{sgn}(\theta)\}$ and $\phi\left(\theta_{J-\frac{1}{2}}\right)=$ $\phi\left(\theta_{J-1-\frac{1}{2}}\right)=\max \{0, \theta\}$. Different choices of limiters $([16],[18])$ can be treated in a similar way. For simplicity here we consider only the minimod method.

Such a method, as it is stated by Harten's theorem ([10]), is TV-stable if $r<1$ and $0 \leqslant \phi(\theta) \leqslant \operatorname{minmod}(2,2 \theta)$. These conditions are clearly satisfied by the scheme (3.9).

\section{Numerical Results and Comparison}

In this section we present the results of numerical experiments by means of the upwind (UWM), the Lax-Wendroff (LWM) and the high resolution method. All methods were implemented in MATLAB programs using double precision arithmetic.

We present the numerical solution of the problem for $f(s)=e^{-s}$. The problem was solved numerically on a uniform grid consisting of $J=20,40,80$, or 160 subintervals, for $r=\frac{1}{2}$, $\lambda=0.5476=\lambda^{*}-0.1<\lambda^{*}=0.6476$ (the value of $\lambda^{*}$ is for this specific form of $f,[17]$ ), and in a time interval $[0, T]$ with $T=10$. The time $T$ is chosen in such a way so that, in all of the following simulations, $\left\|U^{N}-U^{N-1}\right\|_{\infty}<10^{-7}$, to assure that the numerical solutions reach the steady state. Also the initial condition was taken to be $u_{0}(x)=u(x, 0)=0$.

We compare the solution at the time level $t_{N}=T$ with the steady state solution $w(x)$ which is known. More specifically the smaller positive root, $\mu$ of the equation $\sqrt{\mu} \ln \left(\lambda / \mu^{2}+1\right)=$ $\lambda$ will determine $w(x)=\underline{w}(x)=\ln \left(\lambda x / \mu^{2}+1\right)$ the lower stable solution of the steady problem in which, starting with zero initial data, we know that the solution of problem (1.1) converges $([15])$. We set $\left\|e^{N}\right\|_{\infty}=\left\|U^{N}-w(x)\right\|_{\infty}$ where $w(x)$ is evaluated at the points $0=x_{0}, x_{1}, \ldots, x_{J}=1$.

Initially we present in Table (T1) the values of the calculated numerical solution at the time $T$ with both methods together with the exact solution and their error. In this experiment $J=160, r=\frac{1}{2}$ and $\Delta t=0.0031$. 
Table (T1)

Calculated values of $U_{h}$ together with the exact solution for $J=160$.

\begin{tabular}{|c|c||c|c||c|c||}
\hline$x$ & Exact & Upwind & UWM error & Lax-Wendroff & LWM error \\
\hline 0.1 & 0.13112200724761 & 0.12982943696673 & $1.2926(-3)$ & 0.13111091697485 & $1.1090(-5)$ \\
0.2 & 0.24702705481565 & 0.24477407267762 & $2.2530(-3)$ & 0.24700780161837 & $1.9253(-5)$ \\
0.3 & 0.35088263653835 & 0.34789418183696 & $2.9885(-3)$ & 0.35085713563715 & $2.5501(-5)$ \\
0.4 & 0.44495981994690 & 0.44139433305167 & $3.5655(-3)$ & 0.44492938370692 & $3.0436(-5)$ \\
0.5 & 0.53094253734067 & 0.52691523080510 & $4.0273(-3)$ & 0.53090809842761 & $3.4439(-5)$ \\
0.6 & 0.61011401350040 & 0.60571090144046 & $4.4031(-3)$ & 0.61007625426600 & $3.7759(-5)$ \\
0.7 & 0.68347461117119 & 0.67876134637587 & $4.7133(-3)$ & 0.68343404251149 & $4.0569(-5)$ \\
0.8 & 0.75181930597843 & 0.74684695435876 & $4.9724(-3)$ & 0.75177631742049 & $4.2989(-5)$ \\
0.9 & 0.81579032767599 & 0.81059926081556 & $5.1911(-3)$ & 0.81574522030464 & $4.5107(-5)$ \\
1 & 0.87591394710144 & 0.87053653743357 & $5.3774(-3)$ & 0.87586696953442 & $4.6978(-5)$ \\
\hline
\end{tabular}

Note that the error of both methods increases as $x$ increases and attains its maximum value at the point $x=1$.

In addition in Figure (1) the numerical solution of the problem with the Lax - Wendroff method, $u(x, t), 0 \leqslant x \leqslant 1,0 \leqslant t \leqslant T$, is plotted against space and time. In this figure $J=20$.

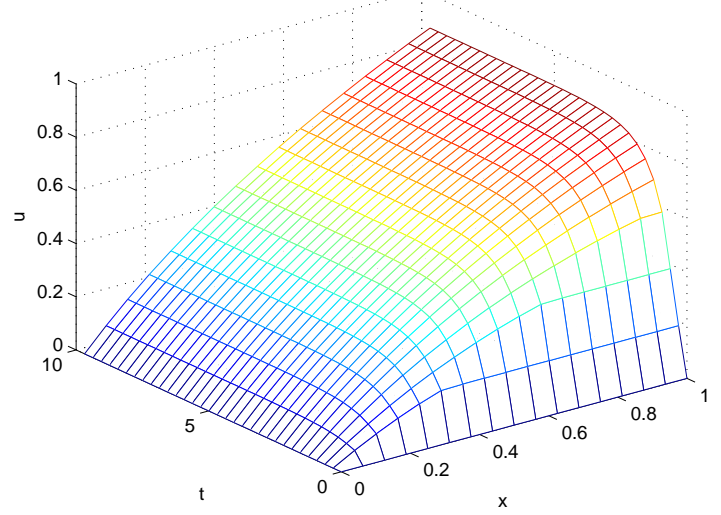

Figure 1. Numerical solution of problem (1.1) with the Lax - Wendroff method, against space and time.

Also in Figure (2a) the maximum in space of the numerical solution, i.e. $u(1, t)$, with both the upwind and Lax - Wendroff method, is plotted against time and in Figure (2b) the profile of the numerical solution at time $T$, again with both methods, is plotted against space, together with the steady state $w(x), 0 \leqslant x \leqslant 1$.

In Table (T2) we present the error, the CPU time, in the brackets the relative CPU time, and the rate of convergence for these two methods. We see that the rate converges to 1 for the upwind method and to 2 for the Lax-Wendrof method. The maximum error of the Lax-Wendroff method is much smaller while the CPU time for the upwind method is lower.

Table (T2).

Rates of convergence of the Upwind and Lax- Wendroff methods.

\begin{tabular}{|c||c|c|c||c|c|c||}
\hline$J$ & $\left\|U^{n}-w_{h}\right\|_{\infty}^{U P}$ & Rate & CPU time (sec) & $\left\|U^{n}-w_{h}\right\|_{\infty}^{L W}$ & Rate & CPU time (sec) \\
\hline 20 & $3.9975(-2)$ & & $0.0492(1)$ & $2.6903(-3)$ & & $0.1886(3.8338)$ \\
40 & $2.0816(-2)$ & 0.9414 & $0.0739(1.5009)$ & $6.9895(-4)$ & 1.9445 & $0.5815(11.8187)$ \\
80 & $1.0635(-2)$ & 0.9689 & $0.1370(2.7827)$ & $1.7930(-4)$ & 1.9628 & $3.9824(80.9441)$ \\
160 & $5.3774(-3)$ & 0.9838 & $0.4757(9.6614)$ & $4.6978(-5)$ & 1.9324 & $27.5828(560.6251)$ \\
\hline
\end{tabular}

In Figure (3) the problem is solved by both the high resolution, with the minimod limiter, method defined by equation (3.8) in (3a) and the Lax-Wendroff method, in (3b). The values 

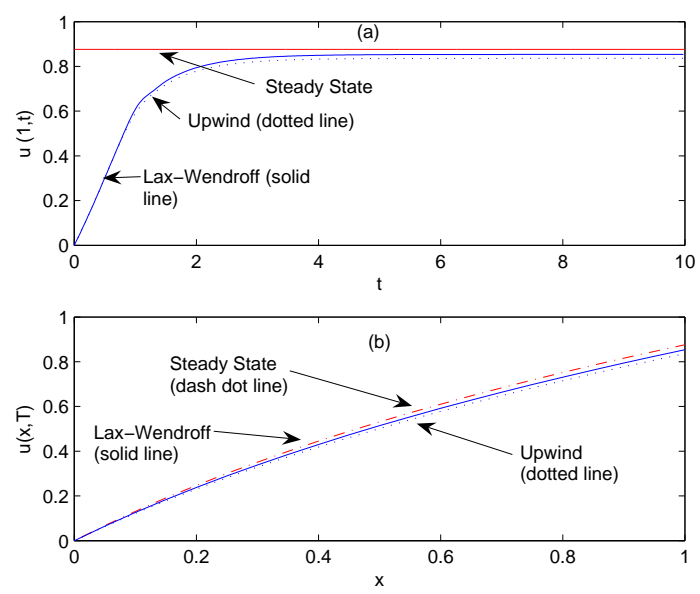

Figure 2. Profiles of the numerical solution of problem (1.1) by the upwind and Lax - Wendroff methods plotted together with the steady state solution. a) $u(1, t), 0 \leqslant t \leqslant T, b) u(x, T), 0 \leqslant x \leqslant 1$.

of the parameters are the same as for the previous simulations but as an initial condition it was taken $u(x, 0)=0$ for $0<x<\frac{1}{4}$ and $x \geqslant \frac{1}{2}$ and $u(x, 0)=1$ for $\frac{1}{4} \leqslant x<\frac{1}{2}, r=.8$ and $\Delta t=0.005$. As it is also stated in [15] discontinuities of the initial condition propagate along characteristics. This can be seen in both Figure (3a) and (3b). More specifically in Figure (3a) produced by the Lax-Wendroff method it can be seen that oscillations appear at the discontinuities, in the direction of characteristics while the high resolution method has much better behaviour with no oscillations. This is more clear in Figure (4) where a profile of
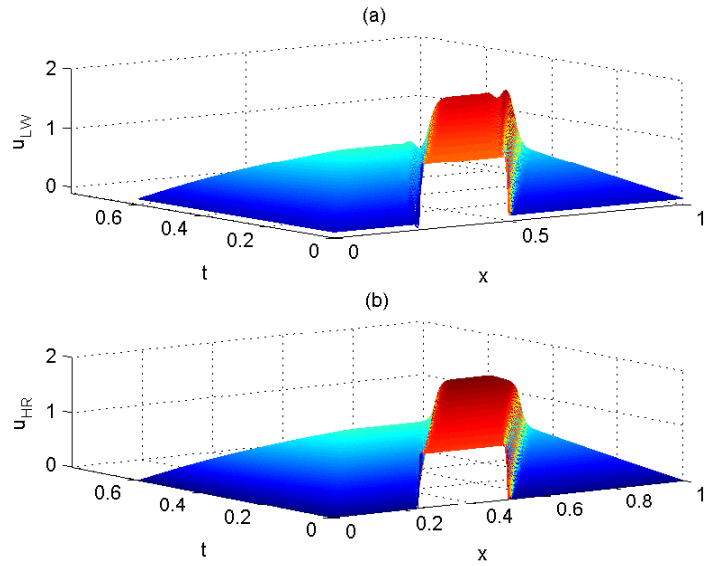

Figure 3. Numerical solution of problem (1.1) with the Lax - Wendroff method (3(a)) and with the high resolution method (3(a)), against space and time for a discontinuous initial condition.

the numerical solutions presented in Figure (3) is plotted with both methods against space at time $t_{0}=0.045$. 


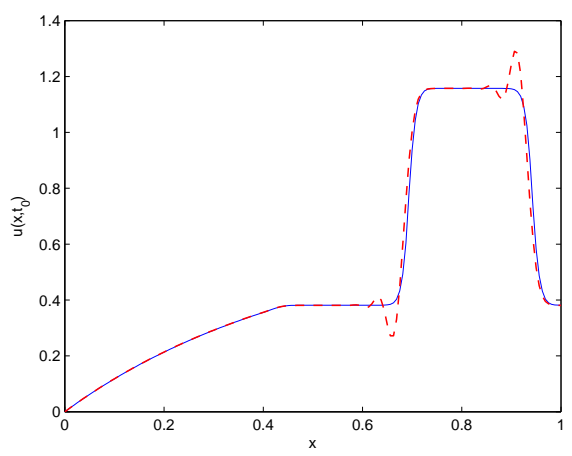

Figure 4. Profiles of the numerical solution of problem (1.1) with both the high resolution (solid line) and the Lax - Wendroff method (dashed line) for $t_{0}=0.045$.

\section{Conclusions}

In the present work an upwind and a Lax - Wendroff scheme are introduced for the solution of a one dimensional non-local problem modelling Ohmic heating of foods. These numerical schemes are studied regarding their consistency, stability and rate of convergence for the cases in which the problem attains a global solution in time. It is found that the upwind scheme is of order $O(\Delta t+\Delta x)$ while the Lax - Wendroff scheme is of order $O\left(\Delta t^{2}+\Delta x^{2}\right)$. Also a high resolution method is introduced which is shown that is total-variation-stable and some numerical experiments are presented in support of the theoretical results.

This work indicates that other finite difference methods can be adapted to this problem having the same order of convergence as for the relevant linear problem, with the absence of source term, as far as an appropriate discretization is used for the non-local term. Moreover, in order to obtain higher order accuracy, higher order terms related with the derivatives of the non-local source term should be included. These numerical methods can serve as a tool for investigating the behaviour of the solution of the problem during blow-up which is a characteristic for many non-local problems as problem (1.1). It is possible that a high resolution method as the one introduced here, together with the relevant theoretical analysis, will give more accurate results in the cases that singularities can be developed during blowup. It is also interesting to investigate similar numerical schemes for generalizations of problem (1.1) such as $u_{t}+(G(u))_{x}=\lambda F(u)$ where the function $G$ may depend on $u$ also in a non-local way $([12,23])$ as well as in parabolic problems of the form $u_{t}=u_{x x}+\lambda F(u)$ $([13,14])$.

Acknowledgements. The author wants to thank Dr. K. Housiadas for having various discussions regarding this work.

\section{References}

[1] L.M. Abia \& J.C. López Marcos, On the numerical integration of non-local terms for age structured population models, Mathematical Biosciences, 157, (1999), 147-167

[2] L.M. Abia, J.C. López Marcos, J. Martínez, On the blow-up time convergence of semidiscretizations of reaction-diffusion equations, Applied numerical Mathematics, 26, (1998), 399-414.

[3] L.M. Abia, J.C. López Marcos, J. Martínez, Blow-up for semidiscretizations of reaction-diffusion equations, Applied numerical Mathematics, 20, (1996), 145-156. 
[4] A.A.P. De Alwis \& P.J. Fryer, Operability of the ohmic heating process: electrical conductivity effects, J. Food Eng., 15, (1992), 21-48.

[5] C.H. Biss, S.A. Coombers \& P.J. Skudder, The development and applications of Ohmic heating for continuous processing of particulate foodstuffs, in Process Engineering in the Food Industry, R.W. Field \& J.A Howell eds, (1989), pp 17-27, Elsevier London.

[6] J.A. Carrillo, On a non-local elliptic equation with decreasing nonlinearity arising in plasma physics and heat conduction. Nonlinear Analysis TMA, 32, (1998), 97-115.

[7] M. Degham, On the Solution of an Initial - Boundary Value Problems that Combines Neumann and Integral Condition for the Wave Equation Numerical Methods for Partial Differential Equations, 21, (2004), $24-40$.

[8] P. Freitas \& M. Grinfeld, Stationary solutions of an equation modelling Ohmic heating, Appl. Math. Lett., Vol. 7, No 3, (1994), 1-6.

[9] P.J. Fryer, A.A.P. De Alwis, E. Koury, A.G.F. Stapley \& L. Zhang, Ohmic processing of solid-liquid mixtures: heat generation and convection effects, J. Food Eng., 18, (1993), 101-125.

[10] A. Harten, High resolution schemes for hyperbolic conservation laws, J. Compute Phys., 49, (1983), $357-393$.

[11] C. Hirota \& K. Ozawa, Numerical method of estimating the blow-up time and rate of the solution of ordinary differential equations - An application to the blow-up problems of partial differential equations, Journal of Computational and Applied Mathematics, Vol. 193, No 2 , (2006), 614-637.

[12] N.I. Kavallaris \& D.E. Tzanetis, Behaviour of a non-local reactive-convective problem with variable velocity in ohmic heating of foods, non-local elliptic and parabolic problems, Banach Center Publications, 66, (2004), 189-198.

[13] A.A. Lacey, Thermal runaway in a non-local problem modelling Ohmic heating. Part I : Model derivation and some special cases, Euro. Jl. Appl. Maths., 6, (1995a), 127-144.

[14] A.A. Lacey, Thermal runaway in a non-local problem modelling Ohmic heating. Part II : General proof of blow-up and asymptotics of runaway, Euro. Jl. Appl. Maths., 6, (1995b), 201-224.

[15] A.A. Lacey, D.E. Tzanetis \& P.M. Vlamos, Behaviour of a non-local reactive convective problem modelling Ohmic heating of foods, Quart. Jl. Mech. Apll. Maths. 5, No 4, (1999), 623-644.

[16] R. J. Leveque, Finite Volume Methods for Hyperbolic problems, Caimbridge University Press, 2002.

[17] C.V. Nikolopoulos, D.E. Tzanetis, Estimates of Blow - up time for a non - local reactive - convective problem modelling ohmic heating of foods, Proceedings of the Edinburgh Mathematical Society, 49, 215239.

[18] P. de Oliveira, J. Santos, On a class of high resolution methods for solving hyperbolic conservation laws with source terms, Applied Nonlinear Analysis, edited by Sequeira et al., Kluver Academic/Plenum Publishers, (1999), 403-416.

[19] C.P. Please , D.W. Schwendeman \& P.S. Hagan, Ohmic heating of foods during aseptic processing, IMA Jl. Maths. Bus. Ind., 5, (1994), 283-301.

[20] P. Skudder \& S. Biss, Aseptic processing of food products using Ohmic heating, The Chemical Engineer, 2, (1987), 26-28.

[21] H.J. Stetter, Analysis of Discretization Methods for Ordinary Differential Equations, Springer, Berlin, 1973

[22] R. Stirling, Ohmic heating - a new process for the food industry, Power Eng. J., 6, (1987), 365.

[23] D.E. Tzanetis \& P.M. Vlamos, A non-local problem modelling ohmic heating of foods with variable thermal conductivity, Nonlinear Analysis : Real World Applications, 2, (2001), 443-454.

[24] L. Zhang \& P. J. Fryer, Models for the electrical heating of solid-liquid food mixtures, Chem. Eng. Sci., 48, (1993), 633-642. 